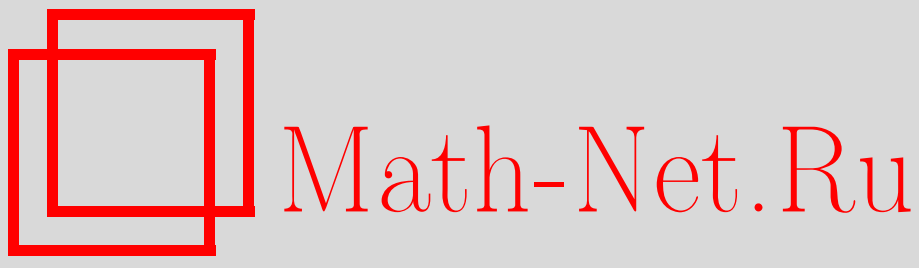

Х. Х. Муртазин, 3. Ю. Фазуллин, Неядерные возмущения дискретных операторов и формулы следов, Матем. сб., 2005, том 196, номер 12, 123-156

DOI: https://doi.org/10.4213/sm1445

Использование Общероссийского математического портала Math-Net.Ru подразумевает, что вы прочитали и согласны с пользовательским соглашением

http://www . mathnet.ru/rus/agreement

Параметры загрузки:

IP: 54.237 .206 .68

26 апреля 2023 г., 13:43:30 
УДК 517.94

\author{
Х.Х. Муртазин, З. Ю. Фазуллин
}

\title{
Неядерные возмущения дискретных операторов и формулы следов
}

\begin{abstract}
В работе получена формула следов для неограниченных дискретных операторов, возмущенных оператором Гильберта-Шмидта, которая может быть названа дискретным аналогом форомулы М.Г. Крейна для ядерных возмущений. Также доказана формула регуляризованного следа типа М.Г. Крейна для возмущений из класса $S^{p}, 2<p \in \mathbb{N}$, для произвольных компактных и относительно компактных возмущений в зависимости от поведения функции распределения спектра невозмущенного оператора на бесконечности.

Библиографйя: 25 названий.
\end{abstract}

\section{Введение}

В настоящей работе изучается вопрос о регуляризованных следах возмущений самосопряженного оператора $T$ с дискретным спектром в сепарабельном гильбертовом пространстве $H$.

Пусть $T$ - самосопряженньй неограниченный дискретный оператор в $H$, $\left\{\lambda_{k}\right\}_{k=-\infty}^{+\infty}-$ собственные числа оператора $T$, пронумерованные в порядке возрастания с учетом их кратностей, $\left\{f_{k}\right\}_{k=-\infty}^{+\infty}-$ ортонормированный базис из собственных функций оператора $T$. Пусть, далее, $V$ - симметрический $T$-компактный оператор, $\left\{\mu_{k}\right\}_{k=-\infty}^{+\infty}-$ собственные числа оператора $L=T+V$, занумерованные в порядке роста с учетом кратностей. Каким условиям должна удовлетворять функция распределения спектра $N(\lambda)$ оператора $T$ в зависимости от возмущения $V$, чтобы сушествовали подпоследовательности натуральных чисел $\left\{n_{m}\right\}_{m=1}^{\infty}$ и $\left\{l_{m}\right\}_{m=1}^{\infty}$ такие, что верна формула

$$
\lim _{m \rightarrow \infty} \sum_{k=-l_{m}}^{n_{m}}\left(\lambda_{k}+\left(V f_{k}, f_{k}\right)-\mu_{k}\right)=0 ?
$$

Отметим, что в случае ядерности $V$ М.Г. Крейном в работе [1] для достаточно широкого класса функций (см. [2]) была получена формула следа

$$
\operatorname{Sp}(\varphi(T+V)-\varphi(T))=\int_{-\infty}^{\infty} \varphi^{\prime}(t) \xi(t) d t
$$

где $\mathrm{Sp}$ - след ядерного оператора, $\xi(t)$ - так называемая функиия спектрального сдвига. Там же установлено равенство

$$
\int_{-\infty}^{\infty} \xi(t) d t=\operatorname{Sp} V
$$


которое означает справедливость формулы $(0.2)$ для $\varphi(t)=t(\mathrm{~cm} .[2 ; \S 3$, п. 4]), откуда для дискретного оператора $T$ из (0.2) вытекает, что

$$
\sum_{k=-\infty}^{+\infty}\left(\mu_{k}-\lambda_{k}\right)=\operatorname{Sp} V
$$

Аналогичный результат для диссипативных ядерных операторов получен в [3], при этом никаких условий на функцию распределения спектра $N(\lambda)$ дискретного оператора $T$ не налагается.

Регуляризованные следы неядерных возмущений дискретных операторов в абстрактной постановке изучались с конца 70-х годов; пионерской здесь была работа [4]. Наиболее сильный результат для конечномерных возмущений был получен в [5], где были охвачены некоторые специальные классы неограниченных возмущений при $N(\lambda)=C \lambda+O\left(\lambda^{p}\right), 0<p<1, C$ - постоянная. Далее, доказательству формулы (0.1) (или более общей формулы, регуляризованной несколькими поправками теории возмущений) были посвящены работы многих математиков, среди которых особо следует отметить работы [6]-[8]. В работе [6] при $N(\lambda)=O\left(\lambda^{p}\right), 0<p<1$, и ограниченном $V$ получены формулы следов для степени $n$ собственных чисел, регуляризованные $l-1$ поправкой теории возмущений, где $l \geqslant n /(1-p)$. В частности, формула $(0.1)$ для ограниченного возмушения была доказана при $0<p \leqslant 1 / 2$, а для возмущения из класса Гильберта-Шмидта - при $0<p \leqslant 1$. В работе [7] формула $(0.1)$ доказана для ограниченных возмущений $V$ при $0<p<2 / 3$ и

$$
\sum_{k=1}^{n} \frac{1}{\lambda_{n+1}-\lambda_{k}}=o(1), \quad n \rightarrow \infty
$$

Наиболее сильньй результат был получен в одной из центральных работ по этой тематике [8], где для доказательства формулы (0.1) для ограниченного, необязательно самосопряженного, возмушения требовалась лишь ядерность резольвенты оператора $T$. Пусть $S^{p}, p>0,-$ множество компактных операторов $V$ таких, что $\|V\|_{p}^{p}=\sum_{k=1}^{\infty} s_{k}^{p}<\infty$, где $s_{k}-s$-числа оператора $V$. В этой же работе [8] для возмушений из класса $S^{p}, 2 \leqslant p \in \mathbb{N}$, была получена формула следов, регуляризованная с $p-1$ поправкой теории возмущений, при условии, что оператор $T$ имеет подпоследовательность собственных чисел $\left\{\lambda_{n_{m}}\right\}_{m=1}^{\infty}$ такую, что $\lambda_{n_{m}+1}-\lambda_{n_{m}} \rightarrow \infty$ при $m \rightarrow \infty$. В частности, оттуда следовала справедливость формулы $(0.1)$ для возмущений из класса Гильберта-Шмидта.

Далее, там же [8; теорема 4] последний результат был усилен, а именно, для компактных возмущений таких, что оператор $V T^{\delta}, \delta \geqslant 0$, может быть продолжен до ограниченного, а оператор $T^{-(1+\delta)}$ ядерньй, была установлена справедливость формулы (0.1).

Отметим также, что в работах [9], [10] изучались обобшения формулы $(0.2)$ для возмущений из класса $S^{2}$.

В [10] доказано, что для каждой функции $\varphi(t)$ из класса

$$
M_{2}=\left\{\varphi: \varphi(x)=\int_{-\infty}^{\infty} e^{i x t} g(t) d t, \quad \int_{-\infty}^{\infty}\left|t^{\nu} g(t)\right| d t<\infty, \quad \nu=0,1,2\right\}
$$


сушествует функция ограниченной вариации $\eta(t)$ :

$$
\bigvee_{-\infty}^{\infty} \eta \leqslant \frac{\|V\|_{2}^{2}}{2}
$$

такая, что справедлива формула

$$
\operatorname{Sp}\left(\varphi(T+V)-\varphi(T)-\left.\frac{d \varphi(T+x V)}{d x}\right|_{x=0}\right)=\int_{-\infty}^{\infty} \varphi^{\prime \prime}(t) d \eta(t) .
$$

В настоящей работе развивается новая техника исследований дискретного спектра в случае самосопряженного возмущения, которая приводит к существенно более сильным результатам абстрактного характера, чем в указанных выше работах.

В первом параграфе рассматриваются возмушения полуограниченного снизу дискретного оператора $T$. Главным результатом п. 1.1 является основная теорема о возмушениях (теорема 1.3), где получено интегральное представление следа резольвентного уравнения возмущенного оператора $L$. Далее, на основе этой теоремы в п. 1.2 получена формула следов для возмущений Гильберта-Шмидта (теорема 1.4). При этом никаких условий на функцию $N(\lambda)$ не налагается, и из этой теоремы вытекают справедливость формулы (0.1), а также формула следов второго порядка для возмущений Гильберта-Шмидта (следствие 1.1). Утверждения следствия 1.1 сильнее соответствующих результатов [6] и теоремы 4 работы [8] для возмушений Гильберта-Шмидта (см. замечание 1.2). Отметим также, что $\varphi(t)=t^{2} \notin M_{2}$, и поэтому следствие 1.1 (см. первую из формул (1.57)) расширяет класс функций $\varphi(t)$ и усиливает результаты вьшеупомянутых работ [9], [10] для дискретных операторов в том смысле, что при $\varphi(t)=t^{2}$ с учетом условия на функцию $\eta(t)$ из $(0.4)$ следует лиш неравенство. Это замечание позволяет заключить, что формула следов для возмущений Гильберта-Шмидта из теорем 1.4, 2.2 может быть названа аналогом формулы М.Г. Крейна (0.3) для ядерных возмушений.

Для компактных и произвольных ограниченных возмущений при доказательстве формулы (0.1) приходится налагать определенные условия на рост функции распределения спектра $N(\lambda)$ невозмущенного оператора $T$. Этому вопросу посвящен п. 1.3. Основным результатом этого пункта является теорема 1.5.

Условие 1) этой теоремы для справедливости формулы (0.1) является довольно универсальньм в том смысле, что из него можно получить утверждения как для компактных (следствие 1.3), ограниченных (замечание 1.6) возмущений, так и для $T$-компактных возмущений (следствие 1.2). Следствие 1.3 сильнее теоремы 4 работы [8] в случае самосопряженных возмушений; утверждение следствия 1.2 сильнее теоремы 5 из [7] и совпадает с утверждением теоремы 1 работы [8] (относительно справедливости формулы (0.1)) для самосопряженных возмущений.

Условия 2)-4) теоремы 1.5 устанавливают порядок роста функции $N(t)$ невозмушенного оператора $T$ в зависимости от конкретных классов возмушений, что является важньм для приложений к дифференциальным операторам.

Особо отметим утверждение п. 3) теоремы 1.5 для ограниченных возмушений, которое перекрывает все ранее известные результаты, в том числе и результаты работы [8] (см. замечание 1.6). Примеры, рассмотренные в п. 3.3, позволяют заключить, что условие 3 ) теоремы 1.5 “ $N(t)=o(t)$ при $t \rightarrow \infty$ ” нельзя ослабить. 
Заметим, что следствие 1.3 (как и теорема 4 работы [8]) предполагает определенные ограничения на скорость стремления к нулю последовательности собственных чисел компактного возмущения $V$ в зависимости от $T\left(V^{2} T^{\delta}\right.$ продолжается до ограниченного оператора, $T^{-(1+\delta)}$ ядерный, $\left.\delta>0\right)$, следовательно, существует компактный оператор $V$, для которого следствие 1.3 неприменимо. Например,

$$
V f=\sum_{k=1}^{\infty} \frac{1}{\ln (k+1)}\left(f, f_{k}\right) f_{k},
$$

где $f_{k}$ - собственные функции оператора $T$, соответствуюшие собственным значениям $\lambda_{k}=k$.

В отличие от этого замечания в п. 2) теоремы 1.5 содержатся лишь ограничения

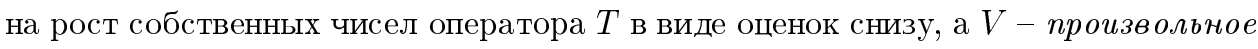
компактное возмущение. Утверждения такого типа ранее нам не были известны.

Отметим, что результаты, относящиеся к возмущениям Гильберта-Шмидта и ограниченным возмушениям, были анонсированы в работе [11].

Во втором параграфе часть результатов, полученных в $\S 1$ для полуограниченных операторов, обобшается на случай неограниченных дискретных операторов.

В $\S 3$ рассматриваются приложения полученных результатов, а также обсуждаются вопросы дальнейшего развития формулы следов.

Авторы выражают благодарность В.Е. Подольскому за полезные обсуждения и замечания.

\section{§1. Возмушения полуограниченных операторов}

1.1. Основная теорема о возмущениях. Пусть $T=T^{*}$ - полуограниченный снизу дискретный оператор в гильбертовом пространстве $H, V$ - симметрический $T$-ограниченњый с нулевой $T$-грањњю оператор в $H$. Тогда известно [12; гл. $\mathrm{V}, \S 4$, п. 4], что $L=T+V$ - самосопряженный полуограниченный снизу дискретный оператор в $H$. Обозначим через $\left\{\lambda_{k}\right\}_{k=1}^{\infty}$ и $\left\{\mu_{k}\right\}_{k=1}^{\infty}$ собственные числа операторов $T$ и $L$ соответственно, пронумерованные в порядке возрастания с учетом их кратностей. Пусть $\left\{f_{k}\right\}_{k=1}^{\infty}-$ базис в $H$ из ортонормированных собственных функций оператора $T$, соответствуюших собственным числам $\lambda_{k}$, т.е.

$$
T f_{k}=\lambda_{k} f_{k}, \quad\left(f_{k}, f_{m}\right)=\delta_{k m} .
$$

Определим операторы проектирования $Q_{n}$, которые будут использоваться в дальнейшем:

$$
Q_{n} h=\sum_{k=1}^{n}\left(h, f_{k}\right) f_{k}
$$

Пусть

$$
G(-\lambda)=(T+\lambda I)^{-1}, \quad R(-\lambda)=(L+\lambda I)^{-1}, \quad \lambda>0 .
$$

Так как операторы $T$ и $L$ полуограничены снизу, то без ограничения обшности можем считать их положительными, поэтому $G(-\lambda)>0$ и $R(-\lambda)>0$. Справедлива 
Лемма 1.1. Пусть оператор $V$ T-ограничен. Тогда для всех $\lambda>0 u$ $\beta \in[0,1]$ оператор $G^{\beta}(-\lambda) V G^{1-\beta}(-\lambda)$ ограничен $и$

$$
\left\|G^{\beta}(-\lambda) V G^{1-\beta}(-\lambda)\right\| \leqslant\|V G(-\lambda)\| .
$$

ДокаЗАТЕЛЬСтво. Для любого $n \in \mathbb{N} Q_{n} V$ - ограниченный оператор в пространстве $H_{n}=Q_{n} H$. Проектор $Q_{n}$ определен в (1.1), причем

$$
\underset{n \rightarrow \infty}{\mathrm{s}-\lim _{n}} Q_{n}=I, \quad H_{n}^{\perp}=\left(I-Q_{n}\right) H .
$$

Отметим, что для достаточно больших $n \in \mathbb{N}$ оператор $Q_{n} V Q_{n}$ отличен от тождественного нуля (если $V \not \equiv 0)$. Действительно, допустим, что $Q_{n} V Q_{n} f=0$ для всех $f \in H$ и всех $n \geqslant n_{0}$. Тогда $V Q_{n} f \in H_{n}^{\perp}$; так как $Q_{n} f=g \in H_{n}$, имеем

$$
V\left(H_{n}\right) \subset H_{n}^{\perp}, \quad n \geqslant n_{0} .
$$

Откуда в силу (1.2) следует, что $\operatorname{ker} V=H$, т.е. $V$ - нулевой оператор.

Пусть $f, \varphi \in H,\|f\|=\|\varphi\|=1$. Для достаточно больших $n$ введем аналитическую функцию

$$
\Phi(z)=\left\|Q_{n} V Q_{n} G(-\lambda)\right\|^{-1}\left(G^{z}(-\lambda) Q_{n} V Q_{n} G^{1-z}(-\lambda) f, \varphi\right) .
$$

Так как

$$
\left\|G(-\lambda) Q_{n} V Q_{n}\right\|=\left\|Q_{n} V Q_{n} G(-\lambda)\right\|,
$$

то для всех $t \in \mathbb{R}$ справедливо $|\Phi(i t)| \leqslant 1,|\Phi(1+i t)| \leqslant 1$. Пользуясь хорошо известной теоремой о трех прямых из теории аналитических функций (см., например, [13; гл. III, §13]), отсюда находим, что при каждом $n \in \mathbb{N}$ и для всех $z, 0 \leqslant \operatorname{Re} z \leqslant 1$,

$$
|\Phi(z)| \leqslant 1 .
$$

Полагая здесь $z=\beta$, получаем неравенство

$$
\left\|G^{\beta}(-\lambda) Q_{n} V Q_{n} G^{1-\beta}(-\lambda)\right\| \leqslant\left\|Q_{n} V Q_{n} G(-\lambda)\right\| .
$$

В силу $T$-ограниченности оператора $V$ имеем

$$
\lim _{n \rightarrow \infty}\left\|Q_{n} V Q_{n} G(-\lambda)\right\|=\|V G(-\lambda)\|,
$$

следовательно, элементарный предельный переход по $n$ в неравенстве (1.3) доказывает лемму.

Покажем, что имеет место следующая

Лемма 1.2. Пусть $V$ - симметрический T-ограниченный оператор с нулевой относительной гранью. Тогда существуют числа $a_{1} u_{2}, 0<a_{1} \leqslant a_{2}$, такие, что при $\lambda \gg 1$ выполняется двойное неравенство

$$
a_{1} G(-\lambda) \leqslant R(-\lambda) \leqslant a_{2} G(-\lambda),
$$

справедливое в смысле квадратичных форм. 
ДокАЗАТЕЛЬСТво. Предыдушая лемма позволяет при $\lambda>0$ записать

$$
T+V+\lambda I=(T+\lambda I)^{1 / 2}\left[I+G^{1 / 2}(-\lambda) V G^{1 / 2}(-\lambda)\right](T+\lambda I)^{1 / 2},
$$

откуда

$$
R(-\lambda)=G^{1 / 2}(-\lambda)\left[I+G^{1 / 2}(-\lambda) V G^{1 / 2}(-\lambda)\right]^{-1} G^{1 / 2}(-\lambda) .
$$

Поскольку оператор $V$-ограничен и имеет относительную грань, равную нулю, то $\|V G(-\lambda)\| \rightarrow 0$ при $\lambda \rightarrow+\infty$ (см., например, [12; гл. $\mathrm{V}, \S 4,(4.4)])$. Следовательно, по лемме 1.1 также

$$
\left\|G^{1 / 2}(-\lambda) V G^{1 / 2}(-\lambda)\right\| \rightarrow 0, \quad \lambda \rightarrow+\infty .
$$

Тогда найдутся такие числа $a_{1}$ и $a_{2}, 0<a_{1} \leqslant a_{2}$, что при $\lambda \gg 1$

$$
a_{1} \leqslant\left\|\left[I+G^{1 / 2}(-\lambda) V G^{1 / 2}(-\lambda)\right]^{-1}\right\| \leqslant a_{2} .
$$

Теперь, так как $G(-\lambda) \geqslant 0$ и $R(-\lambda) \geqslant 0$ при $\lambda>0$, из (1.6), (1.7) для любых $u \in H$ получаем

$$
\begin{aligned}
a_{1}(G(-\lambda) u, u) & =a_{1}\left\|G^{1 / 2}(-\lambda) u\right\|^{2} \leqslant(R(-\lambda) u, u) \\
& \leqslant a_{2}\left\|G^{1 / 2}(-\lambda) u\right\|^{2}=a_{2}(G(-\lambda) u, u) .
\end{aligned}
$$

Лемма доказана.

Введем операторы

$$
K_{0}(\lambda)=G(-\lambda) V G(-\lambda) V G(-\lambda), \quad K(\lambda)=G(-\lambda) V R(-\lambda) V G(-\lambda) .
$$

Ниже нам потребуются условия ядерности операторов $K_{0}(\lambda)$ и $K(\lambda)$. Поскольку $K_{0}(\lambda)$ неотрицателен (при $\lambda>0$ ), то известно (см., например [13; гл. III, $\left.\S 8\right]$ ), что $K_{0}(\lambda)$ ядерный тогда и только тогда, когда для любого ортонормированного базиса $\left\{\varphi_{k}\right\}_{k=1}^{\infty}$ выполняется

$$
\sum_{k=1}^{\infty}\left(K_{0}(\lambda) \varphi_{k}, \varphi_{k}\right)<\infty
$$

причем сумма этого ряда равна спектральному следу $\operatorname{Sp} K_{0}(\lambda)$. Так что оператор $K_{0}(\lambda)$ ядерный (при $\lambda>0$ ), если

$$
\operatorname{Sp} K_{0}(\lambda) \stackrel{\text { def }}{=} \sum_{k=1}^{\infty}\left(K_{0}(\lambda) f_{k}, f_{k}\right)=\sum_{k=1}^{\infty} \sum_{m=1}^{\infty} \frac{\left|\left(V f_{k}, f_{m}\right)\right|^{2}}{\left(\lambda_{k}+\lambda\right)^{2}\left(\lambda_{m}+\lambda\right)}<\infty .
$$

\section{Справедлива}

Лемма 1.3. При $\lambda \gg 1$ оператор $K(\lambda)$ ядерньй тогда и только тогда, когда $K_{0}(\lambda)$ ядерный, причем справедливо

$$
a_{1} \operatorname{Sp} K_{0}(\lambda) \leqslant \operatorname{Sp} K(\lambda) \leqslant a_{2} \operatorname{Sp} K_{0}(\lambda),
$$

əде $\operatorname{Sp} K_{0}(\lambda)$ определяется формулой $(1.8), 0<a_{1} \leqslant a_{2}$. 
ДоКАЗАТЕЛЬСтво. Для доказательства леммы достаточно установить справедливость неравенств (1.9).

Пусть $\left\{\varphi_{k}\right\}$ - ортонормированный базис в $H$. Тогда из определений операторов $K_{0}(\lambda), K(\lambda)$ и леммы 1.2 имеем, что

$$
a_{1}\left(K_{0}(\lambda) \varphi_{k}, \varphi_{k}\right) \leqslant\left(K(\lambda) \varphi_{k}, \varphi_{k}\right) \leqslant a_{2}\left(K_{0}(\lambda) \varphi_{k}, \varphi_{k}\right),
$$

откуда с учетом условий леммы вытекает справедливость неравенств (1.9).

Для дальнейших исследований введем операторы

$$
T_{n}=Q_{n} T Q_{n}, \quad V_{n}=Q_{n} V Q_{n}, \quad L_{n}=T_{n}+V_{n},
$$

где проектор $Q_{n}$ определяется формулой (1.1). Эти операторы будем рассматривать как операторы в пространстве $H_{n}=Q_{n} H$. Через $\sigma\left(L_{n}\right)=\left\{\mu_{k}^{(n)}\right\}_{k=1}^{n}$ обозначим спектр оператора $L_{n}$, причем $\mu_{k}^{(n)} \leqslant \mu_{k+1}^{(n)}$. Имеет место

Лемма 1.4. Для всех $t \in K(K-$ компакт в $\mathbb{R})$ справедливь следующие неравенства:

$$
\begin{gathered}
0 \leqslant \rho(t) \stackrel{\text { def }}{=} \sum_{\lambda_{k}<t}\left[\lambda_{k}+\left(V f_{k}, f_{k}\right)-\mu_{k}\right], \\
0 \leqslant \rho_{n}(t) \stackrel{\text { def }}{=} \sum_{\lambda_{k}<t}\left[\lambda_{k}+\left(V f_{k}, f_{k}\right)-\mu_{k}^{(n)}\right],
\end{gathered}
$$

причем $\rho_{n}(t)=0$ при $t \geqslant \lambda_{n}, \rho_{n}(t) \leqslant \rho_{n+1}(t) u \lim _{n \rightarrow \infty} \rho_{n}(t)=\rho(t)$.

ДокАЗАТЕЛЬСтво. Из принципа минимакса [14; гл. ХІІІ, $\S 2]$ следует, что

$$
\mu_{k}^{(n+1)} \leqslant \mu_{k}^{(n)}, \quad \mu_{k} \leqslant \mu_{k}^{(n)}, \quad k=1, \ldots, n .
$$

Далее, так как $L_{n}$ - конечномерный оператор, его спектральный след совпадает с матричным следом:

$$
\sum_{k=1}^{n} \mu_{k}^{(n)}=\sum_{k=1}^{n}\left(L_{n} f_{k}, f_{k}\right)=\sum_{k=1}^{n}\left[\lambda_{k}+\left(V f_{k}, f_{k}\right)\right] .
$$

Откуда в силу неравенства (1.13) имеем

$$
0 \leqslant \sum_{k=1}^{n}\left(\mu_{k}^{(n)}-\mu_{k}\right)=\sum_{\lambda_{k} \leqslant \lambda_{n}}\left[\lambda_{k}+\left(V f_{k}, f_{k}\right)-\mu_{k}\right]=\rho\left(\lambda_{n}+0\right)
$$

для любого $n \in \mathbb{N}$. Следовательно, так как

$$
\rho(t)=\text { const }, \quad t \in\left[\lambda_{k}, \lambda_{k+1}\right),
$$

для всех $t \in K(K-$ компакт в $\mathbb{R})$ справедливо неравенство (1.11). 
Теперь из равенства (1.14) и неравенства (1.13) согласно определению функции $\rho_{n}(t)$ получаем, что

$$
0=\sum_{\lambda_{k} \leqslant \lambda_{n}}\left[\lambda_{k}+\left(V f_{k}, f_{k}\right)-\mu_{k}^{(n)}\right]=\rho_{n}\left(\lambda_{n}+0\right) \leqslant \rho_{n+1}\left(\lambda_{n}+0\right) .
$$

Откуда заключаем, что для всех $t \in K$ справедливо $0 \leqslant \rho_{n}(t) \leqslant \rho_{n+1}(t)$ и $\rho_{n}(t)=0$ при $t>\lambda_{n}$. Далее, согласно построению

$$
\lim _{n \rightarrow \infty} L_{n} f=L f, \quad f \in \mathscr{D}(T) .
$$

Поэтому из неравенства (1.13) следует, что $\mu_{k}^{(n)}$, монотонно убывая, стремятся к $\mu_{k}$ при $n \rightarrow \infty$, следовательно,

$$
\rho_{n}(t) \nearrow \rho(t), \quad n \rightarrow \infty
$$

Лемма доказана.

ЗАмечАнИЕ 1.1. Неравенство (1.11) было получено в работе [15].

Пусть $G_{n}(-\lambda)=\left(T_{n}+\lambda I\right)^{-1}$ и $R_{n}(-\lambda)=\left(L_{n}+\lambda I\right)^{-1}$ - резольвенты операторов $T_{n}$ и $L_{n}\left(\right.$ см. (1.10)). Тогда из определений операторов $T_{n}, L_{n}$ и $Q_{n}$ легко следует, что

$$
G_{n}(-\lambda)=Q_{n} G(-\lambda)=G(-\lambda) Q_{n}, \quad R_{n}(-\lambda)=Q_{n} R(-\lambda)=R(-\lambda) Q_{n} .
$$

Покажем, что имеет место

ЛЕмма 1.5. При $n \rightarrow \infty$

$$
\begin{array}{ll}
\left\|G(-\lambda)-G_{n}(-\lambda)\right\| \rightarrow 0, & \lambda>0, \\
\left\|R(-\lambda)-R_{n}(-\lambda)\right\| \rightarrow 0, & \lambda \gg 1 .
\end{array}
$$

ДокАЗАТЕЛЬСтво. Пусть $f \in H$ и $\|f\|=1$, тогда (1.16) следует из неравенства

$$
\left\|\left(G(-\lambda)-G_{n}(-\lambda)\right) f\right\|^{2} \leqslant\left(\lambda_{n+1}+\lambda\right)^{-2} \sum_{k=n+1}^{\infty}\left|\left(f, f_{k}\right)\right|^{2}<\lambda_{n+1}^{-2}\|f\|^{2}=\lambda_{n+1}^{-2}
$$

Для доказательства (1.17) в силу $T$-ограниченности оператора $V$ с нулевой относительной гранью заметим, что при $\lambda \gg 1$

$$
\|G(-\lambda) V\|<1, \quad\|R(-\lambda) V\|<1 .
$$

Далее, учитывая (1.15), имеем

$$
\begin{gathered}
R_{n}(-\lambda)+G(-\lambda) V R_{n}(-\lambda)=\left[G(-\lambda)-G_{n}(-\lambda)\right] V R_{n}(-\lambda)+G_{n}(-\lambda), \\
R(-\lambda)+G(-\lambda) V R(-\lambda)=G(-\lambda) .
\end{gathered}
$$

Теперь, вычитая из первого равенства второе, получаем

$$
R_{n}(-\lambda)-R(-\lambda)=(I+G(-\lambda) V)^{-1}\left[G(-\lambda)-G_{n}(-\lambda)\right]\left(V R_{n}(-\lambda)-I\right)
$$

Из (1.16) и последнего соотношения с учетом ограниченности операторов $(I+$ $G(-\lambda) V)^{-1}$ и $\left(V R_{n}(-\lambda)-I\right)$ при $\lambda \gg 1$ (см. (1.18)) вытекает справедливость соотношения (1.17).

Лемма доказана.

Покажем, что имеет место основная теорема этого параграф̆а. 
ТЕОРема 1.1. Пусть оператор $K_{0}(\lambda)$ ядерный, т.е. выполнено (1.8). Тогда при $\lambda \gg 1$ справедливо тождество

$$
2 \int_{0}^{\infty} \frac{\rho(t) d t}{(t+\lambda)^{3}}+\sum_{k=1}^{\infty} \frac{\left(\mu_{k}-\lambda_{k}\right)^{2}}{\left(\lambda_{k}+\lambda\right)^{2}\left(\mu_{k}+\lambda\right)}=\operatorname{Sp} K(\lambda) .
$$

ДоКАЗАТЕЛЬСТВО. Положим

$$
K_{n}(\lambda)=G_{n}(-\lambda) V_{n} R_{n}(-\lambda) V_{n} G_{n}(-\lambda) .
$$

Тогда из резольвентного тождества

$$
R_{n}(-\lambda)-G_{n}(-\lambda)+G_{n}(-\lambda) V_{n} G_{n}(-\lambda)=K_{n}(\lambda),
$$

замечая, что

$$
\begin{gathered}
\operatorname{Sp} G_{n}(-\lambda)=\sum_{k=1}^{n}\left(\lambda_{k}+\lambda\right)^{-1}, \quad \operatorname{Sp} R_{n}(-\lambda)=\sum_{k=1}^{n}\left(\mu_{k}^{(n)}+\lambda\right)^{-1} \\
\operatorname{Sp} G_{n}(-\lambda) V_{n} G_{n}(-\lambda)=\sum_{k=1}^{n} \frac{\left(V f_{k}, f_{k}\right)}{\left(\lambda_{k}+\lambda\right)^{2}} \\
\frac{1}{\mu_{k}^{(n)}+\lambda}=\frac{1}{\lambda_{k}+\lambda}+\frac{\lambda_{k}-\mu_{k}^{(n)}}{\left(\lambda_{k}+\lambda\right)\left(\mu_{k}^{(n)}+\lambda\right)}
\end{gathered}
$$

получаем

$$
\sum_{\lambda_{k} \leqslant \lambda_{n}} \frac{\lambda_{k}+\left(V f_{k}, f_{k}\right)-\mu_{k}^{(n)}}{\left(\lambda_{k}+\lambda\right)^{2}}+\sum_{k=1}^{n} \frac{\left(\mu_{k}^{(n)}-\lambda_{k}\right)^{2}}{\left(\lambda_{k}+\lambda\right)^{2}\left(\mu_{k}^{(n)}+\lambda\right)}=\operatorname{Sp} K_{n}(\lambda) .
$$

Покажем, что $\lim _{n \rightarrow \infty} \operatorname{Sp} K_{n}(\lambda)=\operatorname{Sp} K(\lambda)$. Так как $K_{0}(\lambda)$ - ядерный оператор, из леммы 1.3 следует, что $\operatorname{Sp} K(\lambda)<\infty$. Далее, учитывая (1.15), имеем

$$
\operatorname{Sp} K_{n}(\lambda)=\sum_{k=1}^{n} \frac{\left(V R_{n}(-\lambda) V f_{k}, f_{k}\right)}{\left(\lambda_{k}+\lambda\right)^{2}}
$$

а из леммы 1.5 следует, что при каждом фиксированном $k \in \mathbb{N}$

$$
\lim _{n \rightarrow \infty}\left(V R_{n}(-\lambda) V f_{k}, f_{k}\right)=\left(V R(-\lambda) V f_{k}, f_{k}\right), \quad V f_{k} \in H .
$$

Так как в силу леммы 1.2

$$
a_{1} G_{n}(-\lambda) \leqslant R_{n}(-\lambda) \leqslant a_{2} G_{n}(-\lambda) \leqslant a_{2} G(-\lambda)
$$

и

$$
\operatorname{Sp} K_{0}(\lambda)=\sum_{k=1}^{\infty} \frac{\left(V G(-\lambda) V f_{k}, f_{k}\right)}{\left(\lambda_{k}+\lambda\right)^{2}}<\infty
$$


то для любого $\varepsilon>0$ сушествует число $N \in \mathbb{N}$ такое, что для всех $n>N$

$$
\sum_{k=N}^{n} \frac{\left(V R_{n}(-\lambda) V f_{k}, f_{k}\right)}{\left(\lambda_{k}+\lambda\right)^{2}} \leqslant a_{2} \sum_{k=N}^{\infty} \frac{\left(V G(-\lambda) V f_{k}, f_{k}\right)}{\left(\lambda_{k}+\lambda\right)^{2}}<\varepsilon
$$

Теперь из (1.21)-(1.23) легко вытекает, что

$$
\lim _{n \rightarrow \infty} \operatorname{Sp} K_{n}(\lambda)=\sum_{k=1}^{\infty} \frac{\left(V R(-\lambda) V f_{k}, f_{k}\right)}{\left(\lambda_{k}+\lambda\right)^{2}}=\operatorname{Sp} K(\lambda)
$$

Итак, для доказательства теоремы достаточно показать, что при $n \rightarrow \infty$ левая часть равенства (1.20) стремится к левой части равенства (1.19). С этой целью введем следующие функции:

$$
\begin{gathered}
n_{0}(t)=k, \quad k \leqslant t<k+1, \\
g_{n}(t)= \begin{cases}\frac{\left(\mu_{k}^{(n)}-\lambda_{k}\right)^{2}}{\left(\lambda_{k}+\lambda\right)^{2}\left(\mu_{k}^{(n)}+\lambda\right)}, & k \leqslant t<k+1,1 \leqslant k \leqslant n ; \\
0, & 0 \leqslant t<1, t \geqslant n+1,\end{cases} \\
g(t)= \begin{cases}\frac{\left(\mu_{k}-\lambda_{k}\right)^{2}}{\left(\lambda_{k}+\lambda\right)^{2}\left(\mu_{k}+\lambda\right)}, & k \leqslant t<k+1,1 \leqslant k \leqslant n ; \\
0, & 0 \leqslant t<1 .\end{cases}
\end{gathered}
$$

Используя свойства функции $\rho_{n}(t)$ из леммы 1.4 и учитьвая, что $\rho_{n}(t)=0$ при $t \geqslant \lambda_{n}$, равенство (1.20) представим в виде

$$
2 \int_{0}^{\infty} \frac{\rho_{n}(t) d t}{(t+\lambda)^{3}}+\int_{0}^{\infty} g_{n}(t) d n_{0}(t)=\operatorname{Sp} K_{n}(\lambda)
$$

Оба интеграла в (1.24) неотрицательны и равномерно ограничены по $n$, так как

$$
\operatorname{Sp} K_{n}(\lambda)<a_{2} \operatorname{Sp} K_{0}(\lambda)
$$

Из леммы 1.4 следует, что $\rho_{n}(t) \nearrow \rho(t)$ при $n \rightarrow \infty$, а при каждом фиксированном $k$ величины $\mu_{k}^{(n)}$, монотонно убывая, стремятся к $\mu_{k}$. Следовательно, при $n \rightarrow \infty$ функции $g_{n}(t)$ сходятся к $g(t)$ поточечно. Применяя теорему Фату-Лебега об ограниченной сходимости (см., например, $[16 ;$ гл. $\mathrm{V}, \S 5$, пп. 4,5$])$ к каждому из интегралов в левой части равенства (1.24), получаем справедливость формулы (1.19).

Теорема доказана.

Лемма 1.6. Пусть оператор $V$ симметричен и Т-ограничен с нулевой относительной гранью. Тогда при $\lambda \gg 1$ имеет место равенство

$$
\operatorname{Sp} K(\lambda)=\operatorname{Sp} K_{0}(\lambda)[1+O(\|G(-\lambda) V\|)]
$$


ДокАЗАТЕЛьство. Из резольвентного тождества

$$
R(-\lambda)=G(-\lambda)-G(-\lambda) V G(-\lambda)+G(-\lambda) V R(-\lambda) V G(-\lambda)
$$

и определений операторов $K(\lambda)$ и $K_{0}(\lambda)$ получаем, что

$$
\operatorname{Sp} K(\lambda)=\operatorname{Sp} K_{0}(\lambda)+\operatorname{Sp} K_{1}(\lambda)
$$

где

$$
K_{1}(\lambda)=G(-\lambda) V[-G(-\lambda) V G(-\lambda)+G(-\lambda) V R(-\lambda) V G(-\lambda)] V G(-\lambda) .
$$

Так как $G(-\lambda) \geqslant 0$ при $\lambda>0$, то имеем

$$
\begin{aligned}
\operatorname{Sp} K_{0}(\lambda)= & \sum_{k=1}^{\infty} \frac{\left(V G(-\lambda) V f_{k}, f_{k}\right)}{\left(\lambda_{k}+\lambda\right)^{2}}=\sum_{k=1}^{\infty} \frac{\left\|G^{1 / 2}(-\lambda) V f_{k}\right\|^{2}}{\left(\lambda_{k}+\lambda\right)^{2}} \\
\operatorname{Sp} K_{1}(\lambda)= & \sum_{k=1}^{\infty}\left\{\left(\left[-G^{1 / 2}(-\lambda) V G^{1 / 2}(-\lambda)+G^{1 / 2}(-\lambda) V R(-\lambda) V G^{1 / 2}(-\lambda)\right]\right.\right. \\
& \left.\left.\times G^{1 / 2}(-\lambda) V f_{k}, G^{1 / 2}(-\lambda) V f_{k}\right)\right\}\left(\lambda_{k}+\lambda\right)^{-2} .
\end{aligned}
$$

Далее, воспользовавшись равенством (1.6), неравенством (1.7), а также леммой 1.1, получаем, что при $\lambda \gg 1$

$$
\begin{aligned}
& \left\|-G^{1 / 2}(-\lambda) V G^{1 / 2}(-\lambda)+G^{1 / 2}(-\lambda) V R(-\lambda) V G^{1 / 2}(-\lambda)\right\| \\
& \leqslant\left\|G^{1 / 2}(-\lambda) V G^{1 / 2}(-\lambda)\right\|+a_{2}\left\|G^{1 / 2}(-\lambda) V G^{1 / 2}(-\lambda)\right\| \\
& \leqslant c_{1}\left\|G^{1 / 2}(-\lambda) V G^{1 / 2}(-\lambda)\right\| \leqslant c_{1}\|G(-\lambda) V\|,
\end{aligned}
$$

где $c_{1}$ - положительная постоянная. Из этой оценки и равенств (1.27) и (1.28) заключаем, что

$$
\left|\operatorname{Sp} K_{1}(\lambda)\right| \leqslant c_{1}\|G(-\lambda) V\| \operatorname{Sp} K_{0}(\lambda)
$$

откуда в силу (1.26) следует (1.25).

Лемма доказана.

С целью преобразования второго слагаемого в левой части равенства (1.19) докажем, что справедлива

ЛЕмма 1.7. В условиях теоремы 1.1 для $\lambda \gg 1$

$$
\sum_{k=1}^{\infty} \frac{\left(\lambda_{k}-\mu_{k}\right)^{2}}{\left(\lambda_{k}+\lambda\right)^{2}\left(\mu_{k}+\lambda\right)}=\sum_{k=1}^{\infty} \frac{\left(\lambda_{k}-\mu_{k}\right)^{2}}{\left(\lambda_{k}+\lambda\right)^{3}}[1+O(\|V G(-\lambda)\|)]
$$


ДокАЗАТЕЛЬСТво. Из (1.5) и леммы 1.1 для $\lambda \gg 1$ получаем оценку

$$
[1-\|V G(-\lambda)\|](T+\lambda) \leqslant L+\lambda \leqslant[1+\|V G(-\lambda)\|](T+\lambda)
$$

в смысле квадратичных форм. Следовательно, для всех $k$

$$
[1-\|V G(-\lambda)\|]\left(\lambda_{k}+\lambda\right) \leqslant\left(\mu_{k}+\lambda\right) \leqslant[1+\|V G(-\lambda)\|]\left(\lambda_{k}+\lambda\right)
$$

и равномерно по $k$

$$
\left(\mu_{k}+\lambda\right)^{-1}=\left(\lambda_{k}+\lambda\right)^{-1}[1+O(\|V G(-\lambda)\|)],
$$

откуда следует (1.29). Лемма доказана.

Теперь из теоремы 1.1 и лемм 1.4, 1.6, 1.7 заключаем, что справедлива

ТЕорема 1.2. Пусть оператор $K_{0}(\lambda)$ ядерный, а $V$ - симметрический T-ограниченный оператор с нулевой относительной гранью. Тогда

$$
2 \int_{0}^{\infty} \frac{\rho(t) d t}{(t+\lambda)^{3}}+\sum_{k=1}^{\infty} \frac{\left(\lambda_{k}-\mu_{k}\right)^{2}}{\left(\lambda_{k}+\lambda\right)^{3}}=\operatorname{Sp} K_{0}(\lambda)[1+O(\|V G(-\lambda)\|)] .
$$

Для дальнейшего нам потребуется интегральное представление $\mathrm{Sp} K_{0}(\lambda)$. Для этого от сплошной нумерации спектра оператора $T$ перейдем к поточечной нумерации:

$$
\bar{\lambda}_{1}<\bar{\lambda}_{2}<\cdots<\bar{\lambda}_{n}<\bar{\lambda}_{n+1}<\cdots .
$$

Пусть $P_{k}$ - ортогональньй проектор на собственное подпространство оператора $T$, соответствуюшее собственному значению $\bar{\lambda}_{k}$, положим

$$
a_{k m}=\operatorname{Sp} P_{k} V P_{m} V P_{k} .
$$

Легко видеть, что для всех $k$ и $m$

$$
a_{m k}=a_{k m}, \quad a_{k m} \geqslant 0 .
$$

Действительно,

$$
\begin{aligned}
a_{k m} & =\operatorname{Sp} P_{k} V P_{m} V P_{k}=\operatorname{Sp} P_{k} V P_{m} V=\sum_{l=1}^{\infty}\left(P_{k} V P_{m} V f_{l}, f_{l}\right) \\
& =\sum_{i=1}^{\nu_{k}} \sum_{j=1}^{\nu_{m}}\left|\left(V f_{k_{i}}, f_{m_{j}}\right)\right|^{2} \geqslant 0
\end{aligned}
$$

так как $P_{k}(\cdot)=\sum_{j=1}^{\nu_{k}}\left(\cdot, f_{k_{j}}\right) f_{k_{j}}$, где $f_{k_{j}}, j=1, \ldots, \nu_{k},-$ базис в подпространстве $P_{k} H$.

В обозначениях (1.32) соотношение (1.8) перепишется в виде

$$
\operatorname{Sp} K_{0}(\lambda)=\sum_{k=1}^{\infty} \sum_{m=1}^{\infty} \frac{a_{k m}}{\left(\bar{\lambda}_{k}+\lambda\right)^{2}\left(\bar{\lambda}_{m}+\lambda\right)} .
$$

Имеет место 
ЛЕмма 1.8. Если $K_{0}(\lambda)$ - ядернъий оператор, то

$$
\operatorname{Sp} K_{0}(\lambda)=2 \int_{0}^{\infty} \frac{\tau(t) d t}{(t+\lambda)^{3}}+\sum_{k=1}^{\infty} \frac{a_{k k}}{\left(\bar{\lambda}_{k}+\lambda\right)^{3}}
$$

әде

$$
\tau(t)=\sum_{\bar{\lambda}_{k}<t} \alpha_{k}, \quad \alpha_{k}=\sum_{m \neq k} \frac{a_{k m}}{\bar{\lambda}_{m}-\bar{\lambda}_{k}}
$$

причем $\tau(t)$ представляется также в виде

$$
\tau(t)=\sum_{\bar{\lambda}_{k}<t} \sum_{\bar{\lambda}_{m} \geqslant t} \frac{a_{k m}}{\bar{\lambda}_{m}-\bar{\lambda}_{k}}
$$

(откуда, в частности, следует, что $\tau(t) \geqslant 0)$.

ДокАЗАТЕльство. При каждом натуральном $N$ имеем

$$
\sum_{k=1}^{N} \sum_{m=1}^{N} \frac{a_{k m}}{\left(\bar{\lambda}_{k}+\lambda\right)^{2}\left(\bar{\lambda}_{m}+\lambda\right)}=\sum_{k=1}^{N} \frac{a_{k k}}{\left(\bar{\lambda}_{k}+\lambda\right)^{3}}+\sum_{k=1}^{N} \frac{\alpha_{k}^{(N)}}{\left(\bar{\lambda}_{k}+\lambda\right)^{2}}
$$

где $\alpha_{k}^{(N)}=\sum_{m=1, m \neq k}^{N} a_{k m}\left(\bar{\lambda}_{m}-\bar{\lambda}_{k}\right)^{-1}$, так как в силу $(1.33)$

$$
\sum_{k=1}^{N} \sum_{m=1, m \neq k}^{N} \frac{a_{k m}}{\left(\bar{\lambda}_{m}-\bar{\lambda}_{k}\right)\left(\bar{\lambda}_{k}+\lambda\right)\left(\bar{\lambda}_{m}+\lambda\right)}=0
$$

Равенство (1.38) перепишется в виде

$$
\sum_{k=1}^{N} \sum_{m=1}^{N} \frac{a_{k m}}{\left(\bar{\lambda}_{k}+\lambda\right)^{2}\left(\bar{\lambda}_{m}+\lambda\right)}=\sum_{k=1}^{N} \frac{a_{k k}}{\left(\bar{\lambda}_{k}+\lambda\right)^{3}}+2 \int_{0}^{\lambda_{N}} \frac{\tau^{(N)}(t)}{(t+\lambda)^{3}} d t
$$

где

$$
\tau^{(N)}(t)=\sum_{\bar{\lambda}_{k}<t} \alpha_{k}^{(N)}=\sum_{\bar{\lambda}_{k}<t} \sum_{t \leqslant \bar{\lambda}_{m} \leqslant \bar{\lambda}_{N}} \frac{a_{k m}}{\bar{\lambda}_{m}-\bar{\lambda}_{k}} .
$$

Заметим, что $\tau^{(N)}(t)$ - ступенчатая функция, причем

$$
\int_{0}^{\bar{\lambda}_{N}} \frac{\tau^{(N)}(t)}{(t+\lambda)^{3}} d t=\sum_{k=1}^{N-1} \tau^{(N)}\left(\bar{\lambda}_{k}+0\right) \int_{\bar{\lambda}_{k}}^{\bar{\lambda}_{k+1}} \frac{d t}{(t+\lambda)^{3}} \leqslant \frac{1}{2} \operatorname{Sp} K_{0}(\lambda)
$$

согласно (1.30). Отсюда видно, что для всех $k$ при $N \rightarrow \infty$ существует конечный предел $\tau^{(N)}\left(\bar{\lambda}_{k}+0\right)$, равный $\tau\left(\bar{\lambda}_{k}+0\right)$ (иначе говоря, функция $\tau(t)$ в формуле $(1.36)$ определена корректно). Теперь легко обосновать предельный переход при $N \rightarrow \infty$ в равенстве (1.39), в результате, учитывая (1.34), получаем (1.35).

Лемма доказана.

Теперь покажем, что справедлива основная теорема о возмущениях. 
Tеорема 1.3. Пусть $V$ - симметричный T-компактный оператор в $H u$ Sp $K_{0}(\lambda)<\infty$. Тогда при $\lambda \gg 1$

$$
\int_{0}^{\infty} \frac{\omega(t)}{(t+\lambda)^{4}} d t=\int_{0}^{\infty} \frac{\nu(t)}{(t+\lambda)^{4}} d t[1+O(\|G(-\lambda) V\|)]
$$

2дe

$$
\begin{aligned}
& \omega(t)=2 \int_{0}^{t} \rho(t) d t+\sum_{\lambda_{k}<t}\left(\lambda_{k}-\mu_{k}\right)^{2}, \\
& \nu(t)=2 \int_{0}^{t} \tau(t) d t+\sum_{\bar{\lambda}_{k}<t} a_{k k} .
\end{aligned}
$$

ДокАЗАТЕЛЬСТво. Поскольку функции $\tau(t), \rho(t)$ и числа $a_{k k},\left(\lambda_{k}-\mu_{k}\right)^{2}$ неотрицательны, применяя интегрирование по частям, в силу $(1.42)(\omega(t)=0$ при $t \leqslant 0)$ имеем, что

$$
2 \int_{0}^{r} \frac{\rho(t)}{(t+\lambda)^{3}} d t+\sum_{\lambda_{k} \leqslant r} \frac{\left(\lambda_{k}-\mu_{k}\right)^{2}}{\left(\lambda_{k}+\lambda\right)^{3}}=\frac{\omega(r)}{(r+\lambda)^{3}}+3 \int_{0}^{r} \frac{\omega(t)}{(t+\lambda)^{4}} d t .
$$

Аналогично в силу $(1.43)(\nu(t)=0$ при $t \leqslant 0)$ получаем

$$
2 \int_{0}^{r} \frac{\nu(t)}{(t+\lambda)^{3}} d t+\sum_{\bar{\lambda}_{k} \leqslant r} \frac{a_{k k}}{\left(\bar{\lambda}_{k}+\lambda\right)^{3}}=\frac{\nu(r)}{(r+\lambda)^{3}}+3 \int_{0}^{r} \frac{\nu(t)}{(t+\lambda)^{4}} d t .
$$

Справедливость (1.41) будет следовать из равенств (1.30) и (1.35), если покажем, что

$$
\lim _{r \rightarrow \infty} \frac{\nu(r)}{(r+\lambda)^{3}}=0, \quad \lim _{r \rightarrow \infty} \frac{\omega(r)}{(r+\lambda)^{3}}=0 .
$$

Проверим справедливость первого соотношения в (1.45). Из соотношения (1.44) заключаем, что его правая часть монотонно возрастает по $r$, поэтому в силу (1.35) для любого конечного $r$ выполнено

$$
\frac{\nu(r)}{(r+\lambda)^{3}}+3 \int_{0}^{r} \frac{\nu(t) d t}{(t+\lambda)^{4}} \leqslant \operatorname{Sp} K_{0}(\lambda)<\infty .
$$

Допустим противное, т.е. что

$$
\lim _{r \rightarrow \infty} \frac{\nu(r)}{(r+\lambda)^{3}}=a>0 .
$$

Тогда при $r \gg 1$

$$
\nu(r) \geqslant(a-\varepsilon)(r+\lambda)^{3}, \quad 0<\varepsilon<\frac{a}{2} .
$$

Следовательно,

$$
\lim _{r \rightarrow \infty} \int_{0}^{r} \frac{\nu(t) d t}{(t+\lambda)^{4}}=+\infty
$$

что противоречит неравенству (1.46). Аналогично доказывается справедливость второго соотношения в (1.45).

Теорема доказана.

Отметим, что если оператор $K_{0}(\lambda)$ ядерный, то из соотношений (1.35), (1.44), (1.45) заключаем, что

$$
f(\lambda)=\operatorname{Sp} K_{0}(\lambda)=3 \int_{0}^{\infty} \frac{\nu(t)}{(t+\lambda)^{4}} d t .
$$


1.2. Формулы следов для возмушений Гильберта-Шмидта. Справедлива

Tеорема 1.4. Oператор $V$ является оператором Гильберта-ШАидта тогда и только тогда, когда $f(\lambda)=O\left(\lambda^{-3}\right)$ при $\lambda \rightarrow+\infty$. При этом имеет место формула следов

$$
2 \int_{0}^{\infty} \rho(t) d t+\sum_{k=1}^{\infty}\left(\mu_{k}-\lambda_{k}\right)^{2}=\operatorname{Sp} V^{2}
$$

ДокАЗАТЕЛЬСТво. Пусть $f(\lambda)=O\left(\lambda^{-3}\right)$. Тогда в силу монотонности $\nu(t)$

$$
f(\lambda) \geqslant 3 \int_{\lambda}^{\infty} \frac{\nu(t)}{(t+\lambda)^{4}} d t \geqslant \frac{\nu(\lambda)}{8 \lambda^{3}}
$$

следовательно,

$$
\sup _{\lambda>0} \nu(\lambda)<\infty .
$$

Так что существует конечный предел

$$
\nu(\infty)=\lim _{t \rightarrow \infty} \nu(t)=2 \int_{0}^{\infty} \tau(t) d t+\sum_{k=1}^{\infty} a_{k k} .
$$

Покажем, что $V$ есть оператор Гильберта-Шмидта и выполнено соотношение

$$
\nu(\infty)=\operatorname{Sp} V^{2} .
$$

Для этого сначала, интегрируя по частям в (1.43), получаем

$$
\nu\left(\bar{\lambda}_{n}+0\right)=2 \int_{0}^{\bar{\lambda}_{n}} \tau(t) d t+\sum_{k=1}^{n} a_{k k}=2 \bar{\lambda}_{n} \tau\left(\bar{\lambda}_{n}+0\right)-2 \sum_{k=1}^{n} \bar{\lambda}_{k} \alpha_{k}+\sum_{k=1}^{n} a_{k k}
$$

где $\alpha_{k}$ определяются второй из формул (1.36). Далее, в силу положительности функции $\tau(t)$ и чисел $a_{k k}$ интеграл и ряд в (1.49) сходятся абсолютно, поэтому из (1.49) и (1.51) заключаем, что

$$
\begin{aligned}
\nu(\infty) & =\lim _{n \rightarrow \infty}\left[2 \int_{0}^{\bar{\lambda}_{n}} \tau(t) d t+\sum_{k=1}^{n} a_{k k}\right] \\
& =\lim _{n \rightarrow \infty}\left[2 \bar{\lambda}_{n} \tau\left(\bar{\lambda}_{n}+0\right)-2 \sum_{k=1}^{n} \bar{\lambda}_{n} \alpha_{k}+\sum_{k=1}^{n} a_{k k}\right],
\end{aligned}
$$

где $\alpha_{k}$ определяются второй из формул (1.36).

Так как $a_{m k}=a_{k m}$, то легко показать, что

$$
2 \bar{\lambda}_{n} \rho\left(\bar{\lambda}_{n}+0\right)-2 \sum_{k=1}^{n} \bar{\lambda}_{k} \alpha_{k}+\sum_{k=1}^{n} a_{k k}=d_{n}^{(1)}+d_{n}^{(2)},
$$


где

$$
d_{n}^{(1)}=\sum_{k=1}^{n} \sum_{m=1}^{n} a_{k m}, \quad d_{n}^{(2)}=2 \sum_{k=1}^{n} \sum_{m=n+1}^{\infty} \frac{\bar{\lambda}_{n}-\bar{\lambda}_{k}}{\bar{\lambda}_{m}-\bar{\lambda}_{k}} a_{m k}
$$

Поскольку $a_{k m} \geqslant 0$, то $d_{n}^{(1)}$ - монотонно не убывающая последовательность и $d_{n}^{(2)} \geqslant 0$. Следовательно, согласно (1.52) и (1.53) сушествует

$$
\lim _{n \rightarrow \infty} d_{n}^{(1)}=\sum_{k=1}^{\infty} \sum_{m=1}^{\infty} a_{m k}<\infty .
$$

Так как

$$
d_{n}^{(2)} \leqslant 2 \sum_{k=1}^{n} \sum_{m=n+1}^{\infty} a_{m k}
$$

то в силу $(1.54)$

$$
\lim _{n \rightarrow \infty} d_{n}^{(2)}=0 .
$$

Таким образом, из (1.52), (1.54), (1.55) заключаем, что

$$
\nu(\infty)=\sum_{k=1}^{\infty} \sum_{m=1}^{\infty} a_{k m}=\lim _{n \rightarrow \infty} \sum_{k=1}^{n} \sum_{m=1}^{\infty} a_{k m}
$$

Остается заметить, что

$$
\sum_{m=1}^{\infty} a_{k m}=\operatorname{Sp} P_{k} \sum_{m=1}^{\infty} V P_{m} V P_{k}=\operatorname{Sp} P_{k} V^{2} P_{k}
$$

Отсюда по хорошо известному утверждению из теории операторов [13; гл. III, §8, лемма 8.1] следует, что $V^{2}$ есть ядерный оператор и

$$
\nu(\infty)=\lim _{n \rightarrow \infty} \operatorname{Sp} \sum_{k=1}^{n} P_{k} V^{2} P_{k}=\operatorname{Sp} V^{2}
$$

что совпадает с (1.50). Отсюда по теореме 1.3 легко заключить, что существует конечньй предел

$$
\omega(\infty)=\lim _{t \rightarrow \infty} \omega(t)=2 \int_{0}^{\infty} \rho(t) d t+\sum_{k=1}^{\infty}\left(\mu_{k}-\lambda_{k}\right)^{2}=\operatorname{Sp} V^{2},
$$

что совпадает с (1.48).

Обратно, если $V$ есть оператор Гильберта-Шмидта, то согласно (1.34)

$$
f(\lambda)=\operatorname{Sp} K_{0}(\lambda) \leqslant \frac{1}{\lambda^{3}} \operatorname{Sp} V^{2} .
$$

Теорема доказана. 
СлЕДСТвИЕ 1.1. Если V - самосопряженный оператор Гильберта-ШШмидта, то существует последовательность натуральных чисел $\left\{n_{k}\right\}_{k=1}^{\infty}$ такая, чmo

$$
\begin{gathered}
\lim _{k \rightarrow \infty} \sum_{m=1}^{n_{k}}\left[\lambda_{m}^{2}+2 \lambda_{m}\left(V f_{m}, f_{m}\right)-\mu_{m}^{2}\right]=\lim _{k \rightarrow \infty} \sum_{m=1}^{n_{k}}\left(V^{2} f_{m}, f_{m}\right)=\operatorname{Sp} V^{2} \\
\lim _{k \rightarrow \infty} \lambda_{n_{k}} \rho\left(\lambda_{n_{k}}+0\right)=\lim _{k \rightarrow \infty} \lambda_{n_{k}} \sum_{j=1}^{n_{k}}\left[\lambda_{j}+\left(V f_{j}, f_{j}\right)-\mu_{j}\right]=0 \\
\lim _{k \rightarrow \infty} \rho\left(\lambda_{n_{k}}+0\right)=\lim _{k \rightarrow \infty} \sum_{j=1}^{n_{k}}\left[\lambda_{j}+\left(V f_{j}, f_{j}\right)-\mu_{j}\right]=0
\end{gathered}
$$

Кроме того, выполнено соотночение

$$
\sum_{k=1}^{\infty}\left(\mu_{k}-\lambda_{k}\right)^{2}<\infty
$$

ДокАЗАТЕЛЬСТво. Для любого натурального $n$ имеем

$$
\begin{aligned}
& \sum_{m=1}^{n}\left[-\mu_{m}^{2}+\lambda_{m}^{2}+2 \lambda_{m}\left(V f_{m}, f_{m}\right)-\left(V^{2} f_{m}, f_{m}\right)\right] \\
& \quad=2 \sum_{m=1}^{n} \lambda_{m}\left[\lambda_{m}+\left(V f_{m}, f_{m}\right)-\mu_{m}\right]-\sum_{m=1}^{n}\left(\mu_{m}-\lambda_{m}\right)^{2}+\sum_{m=1}^{n}\left(V^{2} f_{m}, f_{m}\right) \\
& \quad=2 \lambda_{n} \rho\left(\lambda_{n}+0\right)-2 \int_{0}^{\lambda_{n}} \rho(s) d s-\sum_{m=1}^{n}\left(\mu_{m}-\lambda_{m}\right)^{2}+\sum_{m=1}^{n}\left(V^{2} f_{m}, f_{m}\right) .
\end{aligned}
$$

Мы здесь воспользовались равенством

$$
\sum_{m=1}^{n} \lambda_{m}\left[\lambda_{m}+\left(V f_{m}, f_{m}\right)-\mu_{m}\right]=\int_{0}^{\lambda_{n}} t d \rho(t) .
$$

Сумма последних трех слагаемых в (1.60) при $n \rightarrow \infty$ стремится к нулю в силу теоремы 1.4. С другой стороны, так как интеграл $\int_{0}^{\infty} \rho(t) d t$ сходится и $\rho(t) \geqslant 0$, заключаем, что сушествует последовательность $\left\{\lambda_{n_{k}}\right\}_{k=1}^{\infty}$ такая, что

$$
\lim _{k \rightarrow \infty} \lambda_{n_{k}} \rho\left(\lambda_{n_{k}}+0\right)=0
$$

т.е. верно второе соотношение в (1.57). Отсюда получаем формулу (1.58) и в силу (1.60) - первое соотношение в (1.57). Сходимость ряда в (1.59) непосредственно вытекает из формулы (1.48).

Следствие доказано. 
ЗАмечАниЕ 1.2. Для возмущений Гильберта-Шмидта полуограниченных снизу дискретных операторов в работе [6] формула (1.58) была доказана в предположении, что

$$
\lambda \sim c n^{\alpha}, \quad \alpha \geqslant 1, \quad c>0 .
$$

В теореме 4 работы [8] формула (1.58) доказана при условиях, что оператор $V T^{\delta}$ продолжается до ограниченного при некотором $\delta>0$ и при этом оператор $T^{-(1+\delta)}$ ядерный. Покажем, что существуют полуограниченный снизу оператор $T$ и возмущение $V$ из класса Гильберта-Шмидта $S^{2}$, к которьм теорема 4 из [8] не применима. Пусть $\lambda_{n}=c n^{1 / \alpha}, \alpha \geqslant 3, c>0,-$ собственные числа оператора $T$ и $V=T^{-\gamma}$. Такой $V$ будет оператором Гильберта-Шмидта, если $2 \gamma=\alpha(1+\varepsilon)$ (где $\varepsilon$ удовлетворяет, например, ограничениям $0<c_{0} \leqslant \varepsilon<1 / 3$ ). Оператор $V T^{\delta}$ ограничен, если $\gamma \geqslant \delta$. Следовательно,

$$
\frac{1}{\alpha}+\frac{\delta}{\alpha} \leqslant \frac{1}{\alpha}+\frac{\alpha(1+\varepsilon)}{2 \alpha} \leqslant \frac{1}{3}+\frac{1}{2}+\frac{\varepsilon}{2}<1
$$

т.е. оператор $T^{-(1+\delta)}$ неядерный.

В нашей работе для самосопряженных возмушений Гильберта-Шмидта в отличие от вышеперечисленных работ ограничения на рост собственных чисел невозмущенного (полуограниченного снизу) оператора сняты.

Соотношения (1.57) и последнее утверждение (1.59) следствия для абстрактных дискретных операторов ранее не были известны.

1.3. Формулы следов для относительно компактных возмущений. Пусть $V$ - ограниченный самосопряженный оператор в гильбертовом пространстве $H$ и $N(t)=\sum_{\bar{\lambda}_{k}<t} \operatorname{dim} P_{k}-$ функция распределения собственных значений оператора $T$.

Для доказательства формулы следов (1.58), когда $V$ - оператор ГильбертаШмидта, на рост функции $N(t)$ мы никаких условий не налагали. То же самое справедливо в случае ядерности оператора $V$ (см., например, [15]). Для получения формул следов для произвольных ограниченных возмущений приходится налагать определенные условия на функцию $N(t)$. С этой целью сначала покажем, что справедлива

Лемма 1.9. Пусть $V=V^{*}$ - произвольный ограниченный оператор в $H$. Тогда имеет место неравенство

$$
\nu(t) \leqslant 3\|V\|^{2} N(t)
$$

В частности, если $V=V^{*} \in S^{p}, 2<p \in \mathbb{N}$, то

$$
\nu(t) \leqslant 3\left(\operatorname{Sp}|V|^{p}\right)^{2 / p}(N(t))^{(p-2) / p},
$$

где $\nu(t)$ определена формулой (1.43). 
ДокАЗАТЕЛЬСТво. Для доказательства (1.61) заметим, что из (1.43), (1.51) и (1.53) имеем

$$
\nu(t)=\sum_{\bar{\lambda}_{k}<t} \sum_{\bar{\lambda}_{m}<t} a_{k m}+2 \sum_{\bar{\lambda}_{k}<t} \sum_{\bar{\lambda}_{m} \geqslant t} \frac{t-\bar{\lambda}_{k}}{\bar{\lambda}_{m}-\bar{\lambda}_{k}} a_{k m} .
$$

Откуда, так как

$$
0<\left(t-\bar{\lambda}_{k}\right)\left(\bar{\lambda}_{m}-\bar{\lambda}_{k}\right)^{-1} \leqslant 1, \quad a_{k m} \geqslant 0,
$$

используя тождество

$$
\sum_{m=1}^{\infty} a_{k m}=\sum_{m=1}^{\infty} \operatorname{Sp} P_{k} V P_{m} V P_{k}=\operatorname{Sp} P_{k} V^{2} P_{k}
$$

и обозначая через $E_{t}=\sum_{\bar{\lambda}_{k}<t} P_{k}$, получаем

$$
\nu(t) \leqslant 3 \sum_{\lambda_{k}<t} \operatorname{Sp} P_{k} V^{2} P_{k}=3 \mathrm{Sp} E_{t} V^{2} E_{t} \leqslant 3\|V\|^{2} \operatorname{Sp} E_{t}=3\|V\|^{2} N(t) .
$$

Докажем теперь (1.62).

Пусть $\Psi_{t}$ - спектральная функция оператора $V$ и $\|f\|=1$, тогда по неравенству Гёльдера имеем

$$
\begin{aligned}
\left(V^{2} f, f\right) & =\int_{\mathbb{R}} t^{2} d\left(\Psi_{t} f, f\right) \\
& \leqslant\left(\int_{\mathbb{R}}|t|^{p} d\left(\Psi_{t} f, f\right)\right)^{2 / p}\|f\|^{1 / q}=\left(|V|^{p} f, f\right)^{2 / p}, \quad \frac{2}{p}+\frac{1}{q}=1 .
\end{aligned}
$$

Далее, из неравенства (1.64) вытекает, что

$$
\nu(t) \leqslant 3 \operatorname{Sp} E_{t} V^{2} E_{t}=3 \sum_{\lambda_{k}<t}\left(V^{2} f_{k}, f_{k}\right),
$$

откуда в силу (1.65) и неравенства Гёльдера получаем справедливость неравенства (1.62).

Лемма доказана.

Основным результатом этого пункта является

Tеорема 1.5. Пусть $T=T^{*}$ - дискретный полуограниченный снизу, а $V$ - симметрический T-компактный операторы в гильбертовом пространстве Н. И пусть выполнено одно из нижеследующих условий:

1) $\sum_{k=1}^{\infty} \frac{\left(V^{2} f_{k}, f_{k}\right)}{\lambda_{k}}<\infty$

2) $V$ - компактный оператор и $N(t)=O(t)$ при $t \rightarrow \infty$;

3) $V$ - ограниченный оператор и $N(t)=o(t)$ nри $t \rightarrow \infty$;

4) $V \in S^{p}, 2<p \in \mathbb{N}, u N(t)=o\left(t^{p /(p-2)}\right)$ npu $t \rightarrow \infty$.

Тогда существует подпоследовательность натуральных чисел $\left\{n_{m}\right\}_{m=1}^{\infty}$ такая, что

$$
\lim _{m \rightarrow \infty} \rho\left(n_{m}+0\right)=\lim _{m \rightarrow \infty} \sum_{k=1}^{n_{m}}\left(\lambda_{k}+\left(V f_{k}, f_{k}\right)-\mu_{k}\right)=0 .
$$

Сначала покажем, что имеет место следуюшая 
ЛЕмма 1.10. Формула (1.66) справедлива, если

$$
f(\lambda)=o\left(\lambda^{-2}\right), \quad \lambda \rightarrow+\infty
$$

әде $f(\lambda)$ определена формулой (1.47).

ДокАЗАТЕЛЬСтво. Так как функция $f(\lambda)$ удовлетворяет соотношению (1.67), а оператор $V$ является $T$-компактным, с учетом равенств (1.41) и (1.47) имеем, что при $\lambda \gg 1$

$$
\int_{0}^{\infty} \frac{\omega(t)}{(t+\lambda)^{4}} d t=o\left(\lambda^{-2}\right) .
$$

Откуда, так как функция $\omega(t)$ монотонна и неотрицательна (см. (1.42)), следует, что

$$
\frac{\omega(\lambda)}{24 \lambda^{3}} \leqslant \int_{\lambda}^{\infty} \frac{\omega(t)}{(t+\lambda)^{4}} d t=o\left(\lambda^{-2}\right) .
$$

Следовательно, из (1.42) вытекает, что при $\lambda \gg 1$

$$
\int_{0}^{\lambda} \rho(t) d t=o(\lambda)
$$

Теперь легко убедиться в справедливости равенства (1.66). Действительно, так как $\rho(t) \geqslant 0$, то предположение о сушествовании числа $c_{0}>0$ такого, что $\rho(t) \geqslant c_{0}$ при всех $t \gg 1$, противоречит (1.68).

Лемма доказана.

ДОКАЗАТЕЛЬСТВО ТЕОРЕМЫ 1.5. Покажем, что При ВЫПолнении одного из условий 1)-4) теоремы имеет место соотношение (1.67). Тогда из леммы 1.10 будет вытекать справедливость утверждений теоремы 1.5 .

Итак, пусть выполнено условие 1). Покажем, что $f(\lambda)=o\left(\lambda^{-2}\right)$ при $\lambda \rightarrow+\infty$. Действительно, используя формулы (1.47) и (1.8) и равенство Бесселя по переменной $m$ в (1.8) и учитывая, что $\lambda_{k}>0$, имеем

$$
f(\lambda) \leqslant \frac{1}{\lambda^{2}} \sum_{k=1}^{\infty} \frac{\left(V^{2} f_{k}, f_{k}\right)}{\left(\lambda_{k}+\lambda\right)} .
$$

Теперь легко видеть, что при $\lambda \rightarrow+\infty$

$$
\sum_{k=1}^{\infty} \frac{\left(V^{2} f_{k}, f_{k}\right)}{\lambda_{k}+\lambda}=o(1),
$$

так как ряд в правой части равенства (1.69) сходится равномерно для всех $\lambda \geqslant 0$ в силу условия 1$)$.

Пусть теперь выполнено условие 2). Так как $V$ - компактный оператор, то существует последовательность конечномерных операторов $V_{1 n}$ такая, что $V=$ $V_{1 n}+V_{2 n}$, причем $\left\|V_{2 n}\right\| \rightarrow 0$ при $n \rightarrow \infty$ (см. [17; гл. IX, лемма 9.11]).

Далее, заметим, что

$$
\left(V f_{k}, f_{m}\right)^{2}=\left(\left(V_{1 n} f_{k}, f_{m}\right)+\left(V_{2 n} f_{k}, f_{m}\right)\right)^{2} \leqslant 2\left(\left(V_{1 n} f_{k}, f_{m}\right)^{2}+\left(V_{2 n} f_{k}, f_{m}\right)^{2}\right) .
$$


Используя это неравенство для оценки правой части в формуле (1.8) и равенство Парсеваля по переменной $m$ для функции $f(\lambda)$ (см. (1.8) и (1.47)), получаем, что

$$
f(\lambda) \leqslant \frac{2}{\lambda}\left(\sum_{k=1}^{\infty} \frac{\left(V_{1 n}^{2} f_{k}, f_{k}\right)}{\left(\lambda_{k}+\lambda\right)^{2}}+\sum_{k=1}^{\infty} \frac{\left(V_{2 n}^{2} f_{k}, f_{k}\right)}{\left(\lambda_{k}+\lambda\right)^{2}}\right) .
$$

Для оценки правой части (1.70) обозначим через $\left\{\varphi_{l}\right\}_{l=1}^{\infty}$ ортонормированный базис из собственных функций, а через $s_{l}$ - собственные числа оператора $V$. Тогда (см. [17; гл. IX, лемма 9.11]) оператор $V_{1 n}$ можно представить в виде $V_{1 n}(\cdot)=$ $\sum_{l=1}^{n} s_{l}\left(\cdot, \varphi_{l}\right) \varphi_{l}$. Следовательно, для первого слагаемого в правой части (1.70), используя равенство Парсеваля по системе $\left\{f_{k}\right\}_{k=1}^{\infty}$, будем иметь оценку

$$
\sum_{k=1}^{\infty} \frac{\left(V_{1 n}^{2} f_{k}, f_{k}\right)}{\left(\lambda_{k}+\lambda\right)^{2}} \leqslant \frac{1}{\lambda^{2}} \sum_{l=1}^{n} s_{l}^{2}
$$

Для второго слагаемого в правой части (1.70) в силу условия 2) получим следующую оценку:

$$
\sum_{k=1}^{\infty} \frac{\left(V_{2 n}^{2} f_{k}, f_{k}\right)}{\left(\lambda_{k}+\lambda\right)^{2}} \leqslant\left\|V_{2 n}\right\|^{2} \int_{0}^{\infty} \frac{N(t)}{(t+\lambda)^{3}} d t \leqslant C\left\|V_{2 n}\right\|^{2} \int_{0}^{\infty} \frac{t}{(t+\lambda)^{3}} d t=\frac{C\left\|V_{2 n}\right\|^{2}}{\lambda}
$$

Покажем теперь, что для любого $\varepsilon>0$ сушествует $\lambda_{\varepsilon}$ такое, что при $\lambda>\lambda_{\varepsilon}$

$$
f(\lambda)<\frac{\varepsilon}{\lambda^{2}},
$$

т.е. выполнено условие (1.67). Действительно, так как $\left\|V_{2 n}\right\| \rightarrow 0$ при $n \rightarrow \infty$, то найдется номер $n_{0}(\varepsilon)$ такой, что

$$
C\left\|V_{2 n}\right\|^{2}<\frac{\varepsilon}{4}, \quad n \geqslant n_{0}(\varepsilon) .
$$

Положим $n=n_{0}(\varepsilon)$ в правой части (1.71). Тогда, так как $\left|s_{l}\right|<C_{1}, l=1, \ldots, n_{0}(\varepsilon)$, имеем

$$
\frac{1}{\lambda} \sum_{l=1}^{n_{0}(\varepsilon)} s_{l}^{2}<\frac{\varepsilon}{4}
$$

для всех $\lambda>\lambda_{\varepsilon}=C_{1}^{2} n_{0}(\varepsilon) / \varepsilon$.

Теперь легко заметить, что из неравенств (1.71)-(1.75) и оценки (1.70) вытекает неравенство (1.73).

Теперь покажем, что если выполнено одно из условий 3$)$ или 4$)$, то $f(\lambda)$ (см. (1.47)) тоже имеет порядок роста $o\left(\lambda^{-2}\right)$ при $\lambda \rightarrow+\infty$. Действительно, поскольку $\nu(t)$ монотонна и неотрищательна, в силу неравенства (1.61) и условия 3 ) при $\lambda \gg 1$ получаем, что

$$
f(\lambda)=3 \int_{0}^{\infty} \frac{\nu(t)}{(t+\lambda)^{4}} d t \leqslant \frac{7}{8} \frac{\nu(\lambda)}{\lambda^{3}}+9\|V\|^{2} \int_{\lambda}^{\infty} \frac{N(t)}{(t+\lambda)^{4}} d t=o\left(\lambda^{-2}\right) .
$$


Аналогично, из неравенства (1.62) и условия 4$)$ при $\lambda \rightarrow \infty$ имеем

$$
f(\lambda) \leqslant \frac{7}{8} \frac{\nu(\lambda)}{\lambda^{3}}+3\left(\operatorname{Sp}|V|^{p}\right)^{2 / p} \int_{0}^{\infty} \frac{o(t)}{(t+\lambda)^{4}} d t=o\left(\lambda^{-2}\right) .
$$

Теорема доказана.

Условие 1) теоремы 1.5 является довольно универсальным в том смысле, что из него следует справедливость формулы (1.66) как для неограниченных $T$-компактных возмушений (следствие 1.2 ниже), так и для ограниченных и компактных возмушений (см. следствие 1.3 и замечание 1.6 ниже). Условия 2)-4) теоремы 1.5 устанавливают порядок роста функции распределения $N(t)$ невозмушенного оператора $T$ в зависимости от конкретных классов возмушений, что важно для приложений к дифференциальньм операторам. В связи с этим особо отметим условие 3 ) (см. замечание 1.6).

СлеДСТвИЕ 1.2. Пусть симметрический оператор $V$ таков, что $\mathscr{D}(T) \subset$ $\mathscr{D}(V)$, и существует число $\beta, 0 \leqslant \beta<1 / 2$, такое, что оператор $V T^{-\beta}$ продолжается до ограниченного, а оператор $T^{-(1-2 \beta)}$ ядерный. Тогда имеет место формула (1.66).

ДокаЗАТЕЛЬСтво. Легко видеть, что оператор $V$ является $T$-компактным. Покажем, что из условий следствия вытекает справедливость п. 1) теоремы 1.5. Действительно, имеем

$$
\sum_{k=1}^{\infty} \frac{\left(V^{2} f_{k}, f_{k}\right)}{\lambda_{k}}=\sum_{k=1}^{\infty} \frac{\left\|V T^{-\beta} f_{k}\right\|^{2}}{\lambda_{k}^{1-2 \beta}} \leqslant C_{0} \sum_{k=1}^{\infty} \frac{1}{\lambda_{k}^{1-2 \beta}}<\infty
$$

в силу ядерности оператора $T^{-(1-2 \beta)}$.

ЗАмечАниЕ 1.3. В работе [10; теорема 5] формула (1.66) была доказана при следуюших более жестких условиях:

a) $\lambda_{k+1}-\lambda_{k} \sim$ const $k^{1 / \alpha-1}, 0<\alpha<1 / 2$;

b) $\|V f\| \leqslant C_{0}\left\|T^{\beta} f\right\|, 0 \leqslant \beta<1 / 2-\alpha$, $\operatorname{ker} T=\{0\}$.

СЛЕДСТВИЕ 1.3. Пусть существует $\delta \geqslant 0$ такое, что оператор $V^{2} T^{\delta}$ nродолжается до ограниченного, а оператор $T^{-(1+\delta)}$ ядерный. Тогда справедлива формула (1.66).

ДоКАЗАТЕЛЬСТво. Из условий следствия легко вытекает справедливость условия п. 1) теоремы 1.5. В самом деле, имеем

$$
\begin{aligned}
\sum_{k=1}^{\infty} \frac{\left(V^{2} f_{k}, f_{k}\right)}{\lambda_{k}} & =\sum_{k=1}^{\infty} \frac{\left(V^{2} T^{\delta} T^{-\delta} f_{k}, f_{k}\right)}{\lambda_{k}}=\sum_{k=1}^{\infty} \frac{\left(V^{2} T^{\delta} f_{k}, f_{k}\right)}{\lambda_{k}^{1+\delta}} \\
& \leqslant \text { const } \sum_{k=1}^{\infty} \frac{1}{\lambda_{k}^{1+\delta}}<\infty
\end{aligned}
$$

ЗАмечАниЕ 1.4. Следствие 1.3 сильнее теоремы 4 работы [8] в случае самосопряженных возмушений $V$. Там для справедливости формулы (1.66) вместо ограниченности $V^{2} T^{\delta}$ требовалась ограниченность $V T^{\delta}$. 
СЛЕДСТВИЕ 1.4. Формула (1.66) имеет место, если выполнено одно из следующих условий:

1) $V \in S^{2}$

2) $V \in S^{p}, p>2, G(z)=(T-z I)^{-1} \in S^{p /(p-2)}, \operatorname{Im} z \neq 0$, здесь $I-$ тождественный оператор.

ДОКАЗАТЕЛЬСТВо. Покажем, что при выполнении одного из условий следствия выполнено условие 1) теоремы 1.5 .

Действительно, если $V$ - оператор Гильберта-Шмидта, в силу того, что $\lambda_{k}>0$, имеем

$$
\sum_{k=1}^{\infty} \frac{\left(V^{2} f_{k}, f_{k}\right)}{\lambda_{k}} \leqslant c_{0} \sum_{k=1}^{\infty}\left\|V f_{k}\right\|^{2}<+\infty .
$$

Пусть теперь вьполнено условие 2). Тогда из неравенства (1.65) и неравенства Гёльдера следует, что

$$
\begin{aligned}
\sum_{k=1}^{\infty} \frac{\left(V^{2} f_{k}, f_{k}\right)}{\lambda_{k}} & \leqslant \sum_{k=1}^{\infty} \frac{\left(|V|^{p} f_{k}, f_{k}\right)^{2 / p}}{\lambda_{k}} \\
& \leqslant\left(\sum_{k=1}^{\infty}\left(|V|^{p} f_{k}, f_{k}\right)\right)^{1 / p_{0}}\left(\sum_{k=1}^{\infty} \frac{1}{\lambda_{k}^{q_{0}}}\right)^{1 / q_{0}}<+\infty
\end{aligned}
$$

где $p_{0}=p / 2,1 / p_{0}+1 / q_{0}=1$, т.е. $q_{0}=p /(p-2)$.

Следствие доказано.

ЗАмечАниЕ 1.5. Условие 4) теоремы 1.5 слабее условия 2) следствия 1.4. Например, если

$$
N(t) \sim \frac{t^{p /(p-2)}}{\ln t}, \quad t \rightarrow+\infty,
$$

условие 4$)$ теоремы выполнено, но резольвента $G(z)$ оператора $T$ не принадлежит классу $S^{p /(p-2)}$.

ЗАмЕчАниЕ 1.6. Формула (1.66) для ограниченных возмущений (не обязательно самосопряженных) в работе [8] (теорема 1) доказана при условии ядерности резольвенты оператора $T$. Из п. 1) нашей теоремы 1.5 , в частности, вытекает достаточность условия ядерности резольвенты оператора $T$ для справедливости формулы (1.66).

Очевидно, что наше условие " $N(t)=o(t)$ при $t \rightarrow \infty$ " из п. 3) теоремы 1.5 слабее условия ядерности резольвенты оператора $T$, так как из условия п. 3 ) нашей теоремы следует, что для справедливости формулы (1.66) достаточно, чтобы выполнялось $1 / \lambda_{n}=o(1 / n)$ при $n \rightarrow \infty$.

\section{§ 2. Неполуограниченные операторы}

Целью настоящего параграфа является обобщение предыдущих результатов на случай неполуоганиченных дискретных операторов.

Пусть $T$ - дискретный самосопряженный оператор в $H$ с множеством собственных чисел $\left\{\lambda_{k}\right\}_{k=-\infty}^{+\infty}$, пронумерованных в неубываюшем порядке с учетом кратностей, причем $\lambda_{0} \geqslant 0, \lambda_{-1}<0 ;\left\{f_{k}\right\}_{k=-\infty}^{+\infty}-$ соответствующий ортонормированный базис из собственных векторов. Для простоты изложения мы ограничимся рассмотрением ограниченных самосопряженных возмушений $V$. 
Вначале нам предстоит получить нумерацию спектра возмущенного оператора $L=T+V$, которая бы соответствовала нумерации спектра невозмушенного оператора $T$. С этой целью для $n, l \in \mathbb{N}$ введем ортогональные проекторы

$$
\begin{aligned}
Q^{(n, l)} h & =\sum_{k=-n}^{l}\left(h, f_{k}\right) f_{k}, & Q^{(n)} h & =\sum_{k=-n}^{\infty}\left(h, f_{k}\right) f_{k}, \\
Q_{1}^{(l)} h & =\sum_{k=0}^{l}\left(h, f_{k}\right) f_{k}, & Q_{2}^{(n)} h & =\sum_{k=-n}^{-1}\left(h, f_{k}\right) f_{k}, \\
Q_{1} h & =\sum_{k=0}^{\infty}\left(h, f_{k}\right) f_{k}, & Q_{2} & =I-Q_{1}
\end{aligned}
$$

и операторы

$$
\begin{aligned}
L^{(n, l)} & =Q^{(n, l)} L Q^{(n, l)}, & L_{k}^{(l)} & =Q_{k}^{(l)} L Q_{k}^{(l)}, \\
L^{(n)} & =Q^{(n)} L Q^{(n)}, & L_{k} & =Q_{k} L Q_{k},
\end{aligned}
$$

рассматриваемые как операторы, действующие соответственно в подпространствах $Q^{(n, l)} H, Q_{k}^{(l)} H, Q^{(n)} H$ и $Q_{k} H$. Если определим ортогональные суммы операторов

$$
L_{0}^{(n, l)}=L_{1}^{(l)} \oplus L_{2}^{(n)}, \quad L_{0}^{(n)}=L_{1} \oplus L_{2}^{(n)}, \quad L_{0}=L_{1} \oplus L_{2},
$$

то будем иметь

$$
L^{(n, l)}=L_{0}^{(n, l)}+\Phi^{(n, l)}, \quad L^{(n)}=L_{0}^{(n)}+\Phi^{(n)}, \quad L=L_{0}+\Phi,
$$

где

$$
\begin{gathered}
\Phi^{(n, l)}=Q_{2}^{(n)} V Q_{1}^{(l)}+Q_{1}^{(l)} V Q_{2}^{(n)}, \quad \Phi^{(n)}=Q_{1} V Q_{2}^{(n)}+Q_{2}^{(n)} V Q_{1} \\
\Phi=Q_{1} V Q_{2}+Q_{2} V Q_{1}
\end{gathered}
$$

Пусть

$$
\begin{array}{lll}
\left\{\mu_{k}^{(n, l)}\right\}_{k=-n}^{l}, & \left\{\mu_{k 1}^{(l)}\right\}_{k=0}^{l}, & \left\{\mu_{k 2}^{(n)}\right\}_{k=-n}^{-1}, \\
\left\{\mu_{k}^{(n)}\right\}_{k=-n}^{\infty}, & \left\{\mu_{k 1}\right\}_{k=1}^{\infty}, & \left\{\mu_{k 2}\right\}_{k=-\infty}^{-1}
\end{array}
$$

- собственные числа операторов $L^{(n, l)}, L_{1}^{(l)}, L_{2}^{(n)}, L^{(n)}, L_{1}$ и $L_{2}$ соответственно, пронумерованные в неубываюшем порядке с учетом кратностей. Очевидно, имеют место утверждения:

1) для каждого $k,-n \leqslant k<\infty, \mu_{k}^{(n, l)} \searrow \mu_{k}^{(n)}$ nри $l \rightarrow \infty$;

2) для каждого $k \geqslant 0 \mu_{k 1}^{(l)} \searrow \mu_{k 1}$ nрu $l \rightarrow \infty$;

3) для каждого $k \leqslant-1 \quad \mu_{k 2}^{(n)} \nearrow \mu_{k 2}$ при $n \rightarrow \infty$. 
Так как согласно вариационному принципу для каждого $l$ при $k=-n,-n+1, \ldots, l$ справедливо $\mu_{k}^{(n+1, l)} \geqslant \mu_{k}^{(n, l)}$, то в результате предельного перехода по $l$ имеем

$$
\mu_{k}^{n+1} \geqslant \mu_{k}^{(n)}, \quad k \geqslant-n
$$

Предельные значения

$$
\mu_{k}=\lim _{n \rightarrow \infty} \mu_{k}^{(n)}, \quad k \in \mathbb{Z},
$$

и задают упомянутую выше, подходяшую для наших целей, нумерацию собственных чисел оператора $L$.

ЛЕмма 2.1. Существуют натуральнье числа $n_{k}, k=1,2$, не зависящие от l и $n$, такие, что для $n \gg 1, l \gg 1$ справедливы утверждения:

1) при $k \geqslant n_{1}$

$$
\mu_{k}^{(n, l)} \geqslant \mu_{k 1}^{(l)}, \quad \mu_{k}^{(n)} \geqslant \mu_{k 1}, \quad \mu_{k} \geqslant \mu_{k 1}
$$

2) $n p u k \leqslant-n_{2}$

$$
\mu_{k}^{(n, l)} \leqslant \mu_{k 2}^{(n)}, \quad \mu_{k}^{(n)} \leqslant \mu_{k 2}^{(n)}, \quad \mu_{k} \leqslant \mu_{k 2} .
$$

Доказательство. Спектральная задача $L^{(n, l)} u=z u$ равносильна спектральной задаче для системы

$$
\left\{\begin{array}{l}
L_{1}^{(l)} u_{1}+Q_{1}^{(l)} V Q_{2}^{(n)} u_{2}=z u_{1} \\
L_{2}^{(n)} u_{2}+Q_{2}^{(n)} V Q_{1}^{(l)} u_{1}=z u_{2}
\end{array}\right.
$$

где $u_{1}=Q_{1}^{(l)} u, u_{2}=Q_{2}^{(n)} u$. Так как

$$
L_{2}^{(n)}=Q_{2}^{(n)} T Q_{2}^{(n)}+Q_{2}^{(n)} V Q_{2}^{(n)}
$$

и $Q_{2}^{(n)} T Q_{2}^{(n)} \leqslant 0$ в смысле квадратичных форм, то $L_{2}^{(n)} \leqslant\|V\| Q_{2}^{(n)}$. Следовательно, при $z>\|V\|$ в подпространстве $Q_{2}^{(n)} H$ существует резольвента $R_{2}^{(n)}(z)=$ $\left(L_{2}^{(n)}-z\right)^{-1}$, причем

$$
R_{2}^{(n)}(z) \leqslant 0
$$

в смысле квадратичных форм. Так что из второго уравнения системы (2.7) следует, что

$$
u_{2}=-R_{2}^{(n)}(z) Q_{2}^{(n)} V Q_{1}^{(l)} u_{1} .
$$

Следовательно, из первого уравнения вытекает, что $u_{1}$ есть решение уравнения

$$
A_{2}^{(n, l)}(z) u_{1}=z u_{1}
$$

где $A_{2}^{(n, l)}(z)=L_{1}^{(n)}-Q_{1}^{(l)} V Q_{2}^{(n)} R_{2}^{(n)}(z) Q_{2}^{(n)} V Q_{1}^{(l)}$. Ясно, что $A_{2}^{(n, l)}(z)$ дифференцируем по $z$ в равномерной операторной топологии и

$$
\frac{d}{d z} A_{2}^{(n, l)}(z)=-Q_{1}^{(l)} V Q_{2}^{(n)}\left[R_{2}^{(n)}(z)\right]^{2} Q_{2}^{(n)} V Q_{1}^{(l)} .
$$


Пусть $\left\{s_{k 2}^{(n, l)}(z)\right\}_{k=0}^{l+1}-$ собственные значения оператора $A_{2}^{(n, l)}(z)$, пронумерованные в порядке роста с учетом кратностей и дифференцируемые по $z$. Легко показать, что если $\left\{f_{k 2}^{(n, l)}(z)\right\}_{k=0}^{l+1}$ - соответствуюшая нормированная последовательность собственных векторов, сильно дифференцируемых по $z$, то из (2.10) следует

$$
\frac{d}{d z} s_{k 2}^{n, l}(z)=-\left(V Q_{2}^{(n)}\left[R_{2}^{(n)}(z)\right]^{2} Q_{2}^{(n)} V f_{k 2}^{(n, l)}(z), f_{k 2}^{(n, l)}(z)\right)
$$

Далее, согласно (2.9) собственные числа оператора $L^{(n, l)}$ определяются как решения уравнений

$$
s_{k 2}^{(n, l)}(z)=z
$$

для всех $k$, для которых $\mu_{k 1}^{(l)}>\|V\|$. Поэтому из (2.8) следует, что в смысле квадратичных форм $L_{1}^{(n)} \leqslant A_{2}^{(n, l)}(z)$ при $z>\|V\|$, так что

$$
\mu_{k 1}^{(l)} \leqslant s_{k 2}^{(n, l)}(z), \quad k=0, \ldots, l .
$$

Ввиду (2.11), (2.13) для таких $k$ уравнения (2.12) имеют единственные решения $\mu_{k}^{(n, l)}$ такие, что $\mu_{k}^{(n, l)} \geqslant \mu_{k 1}^{(l)}$. Это доказывает первое неравенство п. 1). Остальные два неравенства этого пункта получим предельньми переходами сначала по $l$ при $l \rightarrow \infty$, затем - по $n$ при $n \rightarrow \infty$. Пункт 2 ) леммы 2.1 доказывается совершенно аналогично.

Лемма доказана.

ЛЕмма 2.2. Пусть возмущение $V$ таково, что

$$
\sum_{\left|\lambda_{k}\right|>0}\left\|V f_{k}\right\|^{2}\left|\lambda_{k}\right|^{-1}<\infty
$$

Тогда существует постоянная а> 0 , не зависящая от l u $n$, такая, что при $n \gg 1, l \gg 1$

$$
\begin{gathered}
\sum_{k=n_{1}}^{l}\left(\mu_{k}^{(n, l)}-\mu_{k 1}^{(l)}\right) \leqslant a, \\
\sum_{k=n_{1}}^{\infty}\left(\mu_{k}^{(n)}-\mu_{k 1}\right) \leqslant a, \\
\sum_{k=-n}^{n_{2}}\left(\mu_{k 2}^{(n)}-\mu_{k}^{(n, l)}\right) \leqslant a .
\end{gathered}
$$

Также имеют место неравенства

$$
\sum_{k=-\infty}^{n_{2}}\left(\mu_{k 2}-\mu_{k}\right) \leqslant a, \quad \sum_{k=n_{1}}^{\infty}\left(\mu_{k}-\mu_{k 1}\right) \leqslant a
$$


ДокАЗАТЕЛЬСТво. Пусть

$$
\begin{gathered}
R^{(n, l)}(z)=\left[L^{(n, l)}-z\right]^{-1}, \quad R_{0}^{(n, l)}(z)=\left[L_{0}^{(n, l)}-z\right]^{-1}, \quad R_{1}^{(l)}(z)=\left[L_{1}^{(l)}-z\right]^{-1} \\
R_{2}^{(n)}(z)=\left[L_{2}^{(n)}-z\right]^{-1}, \quad R(z)=(L-z)^{-1} \\
R_{0}(z)=\left(L_{0}-z\right)^{-1}, \quad R_{k}(z)=\left(L_{k}-z\right)^{-1}, \quad k=1,2 .
\end{gathered}
$$

Согласно (2.3), (2.4) имеем

$$
\begin{aligned}
R^{(n, l)}(z)-R_{0}^{(n, l)}(z)= & -R_{0}^{(n, l)} \Phi^{(n, l)} R_{0}^{(n, l)}(z) \\
& +R_{0}^{(n, l)}(z) \Phi^{(n, l)} R^{(n, l)}(z) \Phi^{(n, l)} R_{0}^{(n, l)}(z)
\end{aligned}
$$

причем

$$
R_{0}^{(n, l)}(z)=G_{0}^{(n, l)}(z)\left[I-\Psi^{(n, l)} R_{0}^{(n, l)}(z)\right]=\left[I-R_{0}^{(n, l)} \Psi^{(n, l)}\right] G_{0}^{(n, l)}(z),
$$

где

$$
\begin{gathered}
\Psi^{(n, l)}=Q_{2}^{(n)} V Q_{2}^{(n)} \oplus Q_{1}^{(l)} V Q_{1}^{(l)}, \quad G_{0}^{(n, l)}(z)=\left(T^{(n, l)}-z\right)^{-1} \\
T^{(n, l)}=T_{2}^{(n)} \oplus T_{1}^{(l)}, \quad T_{k}^{(s)}=Q_{k}^{(s)} T Q_{k}^{(s)}, \quad k=1,2 .
\end{gathered}
$$

Легко показать существование чисел $z_{1}>0$ и $\delta>0$, не зависяших от $n$ и $l$, таких, что при всех $n \gg 1, l \gg 1$ для всех операторов, заданных в (2.3), (2.4), расстояние от спектров до точки $z_{1}$ больше или равняется $\delta$. Зафиксировав $z_{1}$, возьмем замкнутый прямоугольный контур $\Gamma^{(n, l)}$, проходящий через точку $z_{1}$ и охватывающий собственные числа операторов $L^{(n, l)}$ и $L_{0}^{(n, l)}$, расположенные правее точки $z_{1}$. Легко видеть, что

$$
\begin{gathered}
\operatorname{Sp} R_{0}^{(n, l)}(z) \Phi^{(n, l)} R_{0}^{(n, l)}(z) \equiv 0 \\
-\frac{1}{2 \pi i} \oint_{\Gamma^{(n, l)}} z \operatorname{Sp}\left[R^{(n, l)}(z)-R_{0}^{(n, l)}(z)\right] d z=\sum_{\mu_{k}^{(n, l)}>z_{1}} \mu_{k}^{(n, l)}-\sum_{\mu_{k 0}^{(n, l)}>z_{1}} \mu_{k 0}^{(n, l)}
\end{gathered}
$$

где $\mu_{k 0}^{(n, l)}$ - собственные числа оператора $L_{0}^{(n, l)}$.

Поэтому из (2.18), (2.20), (2.21) следует, что

$$
\begin{aligned}
& \sum_{\mu_{k}^{(n, l)}>z_{1}} \mu_{k}^{(n, l)}-\sum_{\mu_{k 0}^{(n, l)}>z_{1}} \mu_{k 0}^{(n, l)} \\
& \quad=-\frac{1}{2 \pi i} \oint_{\Gamma^{(n, l)}} z \operatorname{Sp} R_{0}^{(n, l)}(z) \Phi^{(n, l)} R^{(n, l)}(z) \Phi^{(n, l)} R_{0}^{(n, l)}(z) d z
\end{aligned}
$$

Очевидно, здесь интегрирование по контуру $\Gamma^{(n, l)}$ можно заменить интегрированием по вертикальной прямой $\left\{z_{1}+i t, \quad t \in \mathbb{R}\right\}$. Тогда правая часть (2.22) равна

$$
-\frac{1}{2 \pi i} \int_{-\infty}^{\infty} t \operatorname{Sp} R_{0}^{(n, l)}\left(z_{1}+i t\right) \Phi^{(n, l)} R^{(n, l)}\left(z_{1}+i t\right) \Phi^{(n, l)} R_{0}^{(n, l)}\left(z_{1}+i t\right) d t
$$


где учтено, что интеграл от $\operatorname{Sp} R_{0}^{(n, l)}(z) \Phi^{(n, l)} R^{(n, l)}(z) \Phi^{(n, l)} R_{0}^{(n, l)}(z)$ равен нулю, причем согласно (2.19) можно записать

$$
\begin{aligned}
\operatorname{Sp} & R_{0}^{(n, l)}\left(z_{1}+i t\right) \Phi^{(n, l)} R^{(n, l)}\left(z_{1}+i t\right) \Phi^{(n, l)} R_{0}^{(n, l)}\left(z_{1}+i t\right) \\
= & \sum_{k=-n}^{l} \frac{1}{\left(\lambda_{k}-z_{1}+i t\right)^{2}}\left(\left[I-\Psi^{(n, l)} R_{0}^{(n, l)}\left(z_{1}+i t\right)\right] \Phi^{(n, l)} R\left(z_{1}+i t\right) \Phi^{(n, l)}\right. \\
& \left.\times\left[I-R_{0}^{(n, l)}\left(z_{1}+i t\right) \Psi^{(n, l)}\right] f_{k}, f_{k}\right)
\end{aligned}
$$

Отметим, что в силу выбора $z_{1}$ имеем

$$
\left\|R_{0}^{(n, l)}\left(z_{1}+i t\right)\right\| \leqslant \frac{1}{\sqrt{\delta^{2}+t^{2}}}, \quad\left\|R^{(n, l)}\left(z_{1}+i t\right)\right\| \leqslant \frac{1}{\sqrt{\delta^{2}+t^{2}}},
$$

$\left|\lambda_{k}-z_{1}\right| \geqslant \delta$ для всех $k$. Поэтому из (2.24), (2.25) следует, что модуль интеграла в (2.22) оценивается сверху величиной

$$
A \sum_{k=-n}^{l}\left\|V f_{k}\right\|^{2} \int_{-\infty}^{\infty} \frac{d t}{\left|\lambda_{k}-z_{1}\right|^{2}+t^{2}}=A \pi \sum_{k=-n}^{l} \frac{\left\|V f_{k}\right\|^{2}}{\left|\lambda_{k}-z_{1}\right|}
$$

где число $A$ не зависит от $n$ и $l$. Отсюда и из (2.22) следует (2.14). Неравенство (2.15) получим из (2.14) предельным переходом по $l \rightarrow \infty$, который можно совершить ввиду справедливости леммы 2.1 и монотонности последовательностей $\left\{\mu_{k}^{(n, l)}\right\}$ и $\left\{\mu_{k 1}^{(l)}\right\}$. Аналогично доказываются неравенства (2.16), (2.17).

Лемма доказана.

ЛЕмма 2.3. Пусть $\left\{\stackrel{\circ}{\mu}_{k}\right\}_{k=-\infty}^{\infty}-$ упорядоченный набор из чисел $\left\{\mu_{k 1}\right\}_{k=0}^{\infty}$ u $\left\{\mu_{k 2}\right\}_{k=-\infty}^{-1}$. Тогда если выполнено условие леммы 2.2, то ряд

$$
\sum_{k=-\infty}^{\infty}\left(\stackrel{\circ}{\mu}_{k}-\mu_{k}\right)
$$

абсолютно сходится и его сумма равна нулю.

ДокАЗАТЕЛЬСТво. Поскольку $\Phi^{(n)}$ - конечномерньй оператор и $\operatorname{Sp} \Phi^{(n)}=0$, то согласно [1] и (2.4)

$$
\sum_{k=-n}^{\infty}\left(\mu_{k}^{(n)}-\mu_{k 0}^{(n)}\right)=0
$$

причем ряд сходится абсолютно, здесь $\left\{\mu_{k 0}^{(n)}\right\}_{k=-n}^{\infty}-$ упорядоченный набор из чисел $\left\{\mu_{k 2}^{(n)}\right\}_{k=-n}^{-1}$ и $\left\{\mu_{k 1}\right\}_{k=0}^{\infty}$, т.е. $\left\{\mu_{k 0}^{(n)}\right\}_{k=-n}^{\infty}-$ спектр оператора $L_{0}^{(n)}$. Для завершения доказательства леммы остается воспользоваться леммами 2.1, 2.2 и монотонностью последовательностей $\left\{\mu_{k}^{(n)}\right\}$ и $\left\{\mu_{k 2}^{(n)}\right\}$ (для предельного перехода в (2.26)).

Лемма доказана.

Теперь мы в состоянии доказать, что справедлива 
ТеОРема 2.1. Пусть выполнено условие леммы 2.2. Тогда существуют две последовательности $\left\{l_{m}\right\}_{m=1}^{\infty} u\left\{n_{m}\right\}_{m=1}^{\infty}$ натуральных чисел такие, что

$$
\lim _{m \rightarrow \infty} \sum_{k=-n_{m}}^{l_{m}}\left[\lambda_{k}+\left(V f_{k}, f_{k}\right)-\mu_{k}\right]=0
$$

ДОКАЗАТЕЛЬСтво. По теореме 1.5 из 11 существуют последовательности натуральных чисел $\left\{l_{m}\right\}_{m=1}^{\infty}$ и $\left\{n_{m}\right\}_{m=1}^{\infty}$ такие, что

$$
\lim _{m \rightarrow \infty} \sum_{k=0}^{l_{m}}\left[\lambda_{k}+\left(V f_{k}, f_{k}\right)-\mu_{k 1}\right]=\lim _{m \rightarrow \infty} \sum_{k=-n_{m}}^{0}\left[\lambda_{k}+\left(V f_{k}, f_{k}\right)-\mu_{k 2}\right]=0 .
$$

Остается заметить, что при $l_{m} \gg 1$ и $n_{m} \gg 1$

$$
\begin{array}{r}
\sum_{k=-n_{m}}^{l_{m}}\left[\lambda_{k}+\left(V f_{k}, f_{k}\right)-\mu_{k}\right]=\sum_{k=0}^{l_{m}}\left[\lambda_{k}+\left(V f_{k}, f_{k}\right)-\mu_{k 1}\right] \\
+\sum_{k=-n_{m}}^{-1}\left[\lambda_{k}+\left(V f_{k}, f_{k}\right)-\mu_{k 2}\right]+\sum_{k=-n_{m}}^{l_{m}}\left(\stackrel{\circ}{\mu}_{k}-\mu_{k}\right),
\end{array}
$$

и воспользоваться леммой 2.3 .

Теорема доказана.

Для возмушений Гильберта-Шмидта имеет место более сильное утверждение. Для формулировки нам потребуется аналог функции $\rho(t)$ из $\S 1$. Положим

$$
\begin{aligned}
\rho(t) & =\sum_{\lambda_{k}<t}\left[\lambda_{k}+\left(V f_{k}, f_{k}\right)-\mu_{k}\right] \\
& =\sum_{\lambda_{k}<t}\left[\lambda_{k}+\left(V f_{k}, f_{k}\right)-\stackrel{\circ}{\mu}_{k}\right]+\sum_{\lambda_{k}<t}\left(\stackrel{\circ}{\mu}_{k}-\mu_{k}\right),
\end{aligned}
$$

где в правой части сходимость первого ряда понимается как сходимость со скобками (вторая сумма абсолютно сходится).

Имеет место

Теорема 2.2. Пусть $V$ - самосопрязсенный оператор Гильберта-ШШмидma. Тогда $\rho(t) \geqslant 0 u$

$$
2 \int_{-\infty}^{\infty} \rho(t) d t+\sum_{k=-\infty}^{\infty}\left(\mu_{k}-\lambda_{k}\right)^{2}=\operatorname{Sp} V^{2}
$$


ДокАЗАТЕЛЬСТво. По теореме 1.4 из $§ 1$ при каждом $n \in \mathbb{N}$ имеем

$$
2 \int_{\lambda_{-n}}^{\infty} \rho_{n}(t) d t+\sum_{k=-n}^{\infty}\left(\mu_{k}^{(n)}-\lambda_{k}\right)^{2}=\operatorname{Sp}\left[Q^{(n)} V Q^{(n)}\right]^{2},
$$

где

$$
\rho_{n}(t)=\sum_{\lambda_{-n} \leqslant \lambda_{k}<t}\left[\lambda_{n}+\left(V f_{k}, f_{k}\right)-\mu_{k}^{(n)}\right] .
$$

В смысле сходимости со скобками $\rho_{n}(t)$ можно представить в виде

$$
\rho_{n}(t)=\sum_{\lambda_{k} \geqslant t}\left[\mu_{k}^{(n)}-\lambda_{k}-\left(V f_{k}, f_{k}\right)\right] .
$$

Из (2.5) и (2.30) получаем при $t \geqslant \lambda_{-n}$

$$
\rho_{n+1}(t)-\rho_{n}(t)=\sum_{\lambda_{k} \geqslant t}\left[\mu_{k}^{(n+1)}-\mu_{k}^{(n)}\right] \geqslant 0 .
$$

Измонотонности $\mu_{k}^{(n)}$ и $\rho_{n}(t)$ непосредственно следует, что имеет место (2.28), если только заметить, что $\rho_{n}(t)$ можно представить в виде

$$
\rho_{n}(t)=\sum_{\lambda_{-n} \leqslant \lambda_{k}<t}\left[\lambda_{k}+\left(V f_{k}, f_{k}\right)-\mu_{k}\right]+\sum_{\lambda_{-n} \leqslant \lambda_{k}<t}\left(\mu_{k}-\mu_{k}^{(n)}\right),
$$

откуда вытекает, что $\lim _{n \rightarrow \infty} \rho_{n}(t)=\rho(t)$, где $\rho(t)$ определена в $(2.27)$.

Теорема доказана.

\section{§3. Примеры}

В этом параграфе мы укажем класс конкретных операторов, к которьм применимы полученные в предыдуших параграфах результаты, рассмотрим один важный пример и обсудим дальнейшее развитие формул следов (см. п. 3.3).

3.1. В силу известной асимптотической формулы Г. Вейля для функции $N(t)$ (см., например, [18; гл. II, §15, теорема 15.2]) теорема 1.5 применима для широкого класса эллиптических самосопряженных дифференциальных операторов на компактном многообразии. Условие 3 ) теоремы 1.5 выполнено для ограниченных возмушений в случае, когда порядок дифференциального оператора больше размерности многообразия. Для произвольных компактных возмущений (условие 2) теоремы 1.5), порядок дифференциального оператора может быть равен, а для возмушений из класса $S^{p}, 2<p \in \mathbb{N}$ (условие 4) теоремы 1.5) порядок дифференциального оператора может быть и меньше размерности многообразия.

3.2. Рассмотрим оператор $T u=(-\Delta)^{s} u(x, y)$ в $L^{2}(K)$, где $\Delta$ - оператор Лапласа задачи Дирихле, $K$ - квадрат $[0, \pi] \times[0, \pi]$. Известно, что $T$ имеет собственные числа $\lambda_{k m}=\left(k^{2}+m^{2}\right)^{s}$ и собственные функции $f_{k m}=(2 / \pi) \sin k x \sin m x$. Пусть $L=T+V, V$ - оператор умножения на вещественную функцию $v(x, y)$ из $W_{2}^{2}(K)$. Тогда для $s>1$ имеем $N(t)=o(t)$ при $t \rightarrow+\infty$, следовательно, по теореме 1.5 имеет место формула (1.66).

Этот результат в 1999 г. был получен в работе [19] для степени $s>93 / 80$, и доказательство опиралось на тонкие результаты теории чисел. В том же году он был анонсирован нами для $s>1$ и вешественных $v(x, y)$ в работе [11], а для комплекснозначных $v(x, y)$ и $s>1$ доказан в работе [8] в 2002 г. 
3.3. В связи с приложениями к дифференциальным операторам особый интерес представляет случай, когда

$$
\varlimsup_{t \rightarrow+\infty} \frac{N(t)}{t}=c>0
$$

В этом случае для произвольного ограниченного возмушения формула (1.66) уже может не выполняться. Более того, функция $\rho(t)$ (см. (1.11)) при $t \rightarrow \infty$ может вообше не иметь определенного предела. Мы здесь проанализируем два примера.

В пространстве $L^{2}(0, \pi)$ рассмотрим оператор

$$
T f=\sum_{k=1}^{+\infty} k\left(f, f_{k}\right) f_{k}, \quad f_{k}(x)=\sqrt{\frac{2}{\pi}} \sin k x .
$$

Пусть $V$ - оператор умножения на вещественную функцию $v(x)$ из класса $W_{1}^{2}(0, \pi)$, $L=T+V, \sigma(L)=\left\{\mu_{k}\right\}_{k=1}^{+\infty}$. Заметим, что здесь выполнено (3.1) при $c=1$. Тогда нами в работе [11] была получена следуюшая формула регуляризованного следа:

$$
\sum_{k=1}^{+\infty}\left(k+\left(V f_{k}, f_{k}\right)-\mu_{k}\right)=\frac{1}{2 \pi} \int_{0}^{\pi} v^{2}(x) d x-\frac{c_{0}^{2}}{2}, \quad c_{0}=\frac{1}{\pi} \int_{0}^{\pi} v(x) d x .
$$

Из формулы следов (3.2) легко вытекает аналог формулы следов ГельфандаЛевитана для оператора $L$ :

$$
\sum_{k=1}^{\infty}\left(k+c_{0}-\mu_{k}\right)=\frac{1}{4}(v(0)+v(\pi))-\frac{c_{0}}{2}+\frac{1}{2 \pi} \int_{0}^{\pi} v^{2}(x) d x-\frac{c_{0}^{2}}{2} .
$$

В качестве второго примера рассмотрим оператор $L$ из п. 3.2 при $s=1$, т.е. оператор Лапласа задачи Дирихле, возмушенный оператором умножения на вещественную функцию $v(x, y)$ из класса $W_{2}^{2}(K)$. Отметим, что для этой задачи выполнено (3.1) при $c=\pi / 4$. В силу тауберовой теоремы типа Келдыша из соотношения (1.41) при $t \rightarrow+\infty$ имеем $\omega(t)=\nu(t)(1+o(1))$. Откуда, воспользовавшись формулами $(1.42),(1.43)$ и равенством Парсеваля по $m$, для первого слагаемого в правой части равенства (1.63) получаем

$$
\begin{aligned}
& 2 \int_{0}^{t} \rho(\tau) d \tau+\sum_{\lambda_{k}<t}\left(\mu_{k}-\lambda_{k}\right)^{2} \\
& \quad=\left(\sum_{\bar{\lambda}_{k}<t} \operatorname{Sp} P_{k} V^{2} P_{k}+\sum_{\bar{\lambda}_{k}<t} \sum_{\bar{\lambda}_{m} \geqslant t}\left(\frac{2\left(t-\bar{\lambda}_{k}\right)}{\bar{\lambda}_{m}-\lambda_{k}}-1\right) a_{k m}\right)(1+o(1)) .
\end{aligned}
$$

Теперь в правой части этого равенства, используя собственные функции

$$
f_{i j}(x, y)=\frac{2}{\pi} \sin i x \sin j y, \quad i^{2}+j^{2}=k=\bar{\lambda}_{k},
$$


оператора Лапласа задачи Дирихле, после несложных, но довольно громоздких вычислений заключаем, что

$$
\begin{gathered}
2 \int_{0}^{t} \rho(\tau) d \tau+\sum_{\lambda_{k}<t}\left(\mu_{k}-\lambda_{k}\right)^{2}=\frac{1}{\pi^{2}} \int_{0}^{\pi} \int_{0}^{\pi} v^{2}(x, y) d x d y N(t)(1+o(1)) \\
\quad=\frac{t}{4 \pi} \int_{0}^{\pi} \int_{0}^{\pi} v^{2}(x, y) d x d y(1+o(1))
\end{gathered}
$$

Из (3.3) видно, что функция $\rho(t)$ при $t \rightarrow+\infty$ имеет сходящиеся частичные пределы. Однако ожидать, что $\rho(t)$ при $t \rightarrow+\infty$ имеет определенный предел, не приходится. Действительно, пусть $v(x, y)=q_{1}(x)+q_{2}(y)$, тогда в уравнении

$$
-\Delta u+v u=\lambda u
$$

переменные разделяются и задача сводится к изучению спектра обыкновенных дифференциальных операторов в $L^{2}(0, \pi)$ :

$$
L_{i} f(t)=-f^{\prime \prime}(t)+q_{i}(t) f(t), \quad f(0)=f(\pi)=0, \quad i=1,2 .
$$

Пусть $\sigma\left(L_{i}\right)=\left\{\mu_{k}^{(i)}\right\}_{k=1}^{\infty}-$ спектр оператора $L_{i}, i=1,2, f_{k}(t)=\sqrt{2 / \pi} \sin k x$ - ортонормированные собственные функции оператора $T f(t)=-f^{\prime \prime}(t), f(0)=$ $f(\pi)=0$. Покажем, что справедливо

УТВЕРЖДЕНИЕ 3.1. Пусть $v(x, y)=q_{1}(x)+q_{2}(y), q_{i} \in W_{1}^{2}(0, \pi)$. Тогда

а) при каждом фиксированном натуральном $z$

$$
\begin{aligned}
\lim _{\mathbb{N} \ni l \rightarrow \infty} \rho\left(l^{2}+z+0\right)= & \sum_{i=1}^{2} \sum_{k=1}^{[\sqrt{z}]}\left(k^{2}+\left(q_{i} f_{k}, f_{k}\right)-\mu_{k}^{(i)}\right) \\
& +\frac{1}{8 \pi}\left(\int_{0}^{\pi} \int_{0}^{\pi} v^{2}(x, y) d x d y-c_{0}^{2}\right)
\end{aligned}
$$

$$
\text { где }[\sqrt{z}]-\text { иелая часть числа } \sqrt{z}, c_{0}=\int_{0}^{\pi} \int_{0}^{\pi} v(x, y) d x d y ;
$$

б) $п р и \mathbb{N} \ni z_{l} \rightarrow \infty, z_{l} \leqslant 2 l+1$,

$$
\lim _{l \rightarrow \infty} \rho\left(l^{2}+z_{l}+0\right)=\frac{1}{8 \pi}\left(\int_{0}^{\pi} \int_{0}^{\pi} v^{2}(x, y) d x d y-c_{0}^{2}\right) .
$$

ДокАЗАТЕЛЬСтво. Заметим, что

$$
\sum_{k=1}^{\infty}\left(k^{2}+\left(q_{i} f_{k}, f_{k}\right)-\mu_{k}\right)=0
$$

и что для $k \gg 1$

$$
\mu_{k}^{(i)}=k^{2}+\left(q_{i} f_{k}, f_{k}\right)+\frac{c_{i}}{k^{2}}+O\left(k^{-4}\right)
$$


где

$$
c_{i}=\frac{1}{4 \pi}\left(\int_{0}^{\pi} q_{i}^{2}(t) d t-\left(\frac{1}{\pi} \int_{0}^{\pi} q_{i}(t) d t\right)^{2}\right), \quad i=1,2
$$

(см., например, [20]). Поэтому, полагая $1 \leqslant m \leqslant l, k=\left[\sqrt{l^{2}+z-m^{2}}\right](z-$ фиксированное натуральное число), имеем

$$
\begin{aligned}
\rho\left(l^{2}+z+0\right)= & \sum_{i=1}^{2} \sum_{m=1}^{l} \sum_{k=1}^{\left[\sqrt{l^{2}+z-m^{2}}\right]}\left(k^{2}+\left(q_{i} f_{k}, f_{k}\right)-\mu_{k}^{(i)}\right) \\
= & \sum_{i=1}^{2}\left\{\sum_{k=1}^{[\sqrt{z}]}\left(k^{2}+\left(q_{i} f_{k}, f_{k}\right)-\mu_{k}^{(i)}\right)\right. \\
& +\sum_{m=1}^{l-1}\left[\sum_{k=1}^{\left[\sqrt{l^{2}+z-m^{2}}\right]}\left(k^{2}+\left(q_{i} f_{k}, f_{k}\right)-\mu_{k}^{(i)}\right)\right\} \\
= & \sum_{i=1}^{2}\left\{\sum_{k=1}^{[\sqrt{z}]}\left(k^{2}+\left(q_{i} f_{k}, f_{k}\right)-\mu_{k}^{(i)}\right)\right. \\
& \left.+\sum_{m=1}^{\infty-1} \sum_{k=\left[\sqrt{l^{2}+z-m^{2}}\right]+1}^{\infty}\left(\frac{c_{i}}{k^{2}}+O\left(k^{-4}\right)\right)\right\} .
\end{aligned}
$$

Откуда следует тождество (3.4), так как легко убедиться, что

$$
\lim _{l \rightarrow \infty} \sum_{m=1}^{l-1} \sum_{k=\left[\sqrt{l^{2}+z-m^{2}}\right]+1}^{\infty}\left(\frac{c_{i}}{k^{2}}+O\left(k^{-4}\right)\right)=\frac{\pi c_{i}}{2} .
$$

Справедливость п. б) утверждения вытекает из соотношения (3.7), если в нем положить $z=z_{l}, 0<z_{l} \leqslant 2 l+1, \mathbb{N} \ni z_{l} \rightarrow \infty$ при $l \rightarrow \infty$, и воспользоваться тождеством (3.6).

Данное утверждение показывает, что изучение поведения $\rho(t)$ при $t \rightarrow \infty$ в общем случае представляет собой весьма трудную математическую задачу. Мы ожидаем, что должны быть справедливы формулы вида (3.4), (3.5). Отметим также, что прослеживается определенная аналогия между формулами (3.2) и (3.5). В связи с этим заметим, что аналогичная формула справедлива для следа возмушения оператора Лапласа-Бельтрами на двумерной сфере $S^{2}$ (при этом в формуле $(3.1) c=1)$, изученного в других терминах в работах [21]-[24], и для следа возмушения двумерного гармонического осциллятора, для которого она получена в работе [25]. 


\section{Список литературы}

1. Крейн М. Г. О формуле следов в теории возмущений // Матем. сб. 1953. Т. 33 (75). № 3. C. 597-626.

2. Бирман М.ШІ, Яфаев Д. Р. Функция спектрального сдвига. Работы М.Г. Крейна и их дальнейшее развитие // Алгебра и анализ. 1992. Т. 4. № 5. С. 1-44.

3. Адамян В. М., Павлов Б. С. Формула следов для диссипативных операторов // Вестн. ЛГУ. Сер. матем. 1979. № 2. С. 5-9.

4. Садовничий B. А., Дубровский В. В. Об одной абстрактной теореме теории возмущений, о формулах регуляризованных следов и о дзета-функции операторов // Дифференц. уравнения. 1977. Т. 13. № 7. С. 1264-1271.

5. Садовничий B. A., Любишкин $B . A$. Конечномерные возмущения дискретных операторов и формула следов // Функц. анализ и его прилож. 1986. Т. 20. № 3. С. 55-65.

6. Дубровский В. В. Формулы регуляризованных следов операторов с компактной резольвентой // Дифференц. уравнения. 1990. Т. 26. № 12. С. 2046-2051.

7. Dostanic $M$. Trace formula for nonnuclear perturbations of selfadjoint operators // Publ. Inst. Math. (Beograd) (N.S.) 1993. V. 54(68). P. 71-79.

8. Садовничий B. А., Подольский B. Е. Следы операторов с относительно компактным возмущением // Матем. сб. 2002. Т. 193. № 2. С. 129-152.

9. Коплиенко Л. С. О формуле следов для возмушений неядерного типа // Сиб. матем. журн. 1984. Т. 25. № 5. С. $62-71$.

10. Dostanic $M$. Traceformulas of Gelfand-Levitan type // Publ. Inst. Math. (Beograd) (N.S.) 1994. V. 55 (69). P. 51-63.

11. Муртазин X.X., Фазуллин З. Ю. О формулах следов для неядерных возмущений // Докл. РАН. 1999. Т. 368. № 4. С. 442-444.

12. Като Т. Теория возмущений линейных операторов. М.: Мир, 1972.

13. Гохберг И. ЦЦ., Крейн М. Г. Введение в теорию линейных несамосопряженных операторов в гильбертовом пространстве. М.: Наука, Физматлит, 1965.

14. Рид M., Саймон Б. Методы современной математической физики. Т. 4. Анализ операторов. М.: Мир, 1982.

15. Гасымов М. Г. О сумме разностей собственных значений двух самосопряженных операторов // Докл. АН СССР. 1963. Т. 150. №6. С. 1202-1205.

16. Колмогоров A.H., Фомин C. В. Элементы теории функций и функционального анализа. М.: Наука, 1981.

17. Данфорд Н., Швари Джс. Линейные операторы. Т. 2. М.: Мир, 1966.

18. Шубин М. А. Псевдодиффференциальные операторы и спектральная теория. М.: Наука, Физматлит, 1978.

19. Дубровский В. В., Пузанкова Е. А. Оценка разности спектральных функций и формулы регуляризованных следов степени оператора Лапласа, заданного на треугольнике или квадрате в $L^{p}, 1 \leqslant p \leqslant 2 / /$ Дифференц. уравнения. 1999. Т. 35. № 4. С. 552-555.

20. Дикий Л. А. Формулы следов для дифференциальных операторов Штурма-Лиувилля // УМH. 1958. Т. 13. №3. С. 111-143.

21. Садовничий В. А., Дубровский В. В. Классическая формула регуляризованного следа для собственных чисел оператора Лапласа-Бельтрами с потенциалом на сфере $S^{2}$ // Докл. АН СССР. 1991. Т. 319. №1. С. 61-62.

22. Подольский В. Е. Формула регуляризованного следа оператора Лапласа-Бельтрами с нечетньм потенциалом на сфере $S^{2} / /$ Матем. заметки. 1994. Т. 56. № 1. С. 71-77.

23. Фазуллин З. Ю. Формула регуляризованного следа для возмущения оператора Лапласа-Бельтрами // Международная конференция по комплексному анализу и смежньм вопросам (2-5 июня 1997, Нижегородский государственный университет). Тезисы докладов. Нижний Новгород: Изд-во ННГУ, 1997. С. 80-81.

24. Садовничий В.А., Фазуллин З. Ю. Формула первого регуляризованного следа для оператора Лапласа-Бельтрами // Дифференц. уравнения. 2001. Т. 37. № 3. С. 402-409.

25. Фазуллин З. Ю., Муртазин X. Х. Регуляризованный след двумерного гармонического осциллятора // Матем. сб. 2001. Т. 192. № 5. С. 87-124.

Башкирский государственнњй университет,

Поступила в редакцию г. Уфpa 31.01 .2005 и 24.05 .2005 